CERN-EP/99-54

16.2. 1999

\title{
Superconducting NbN Microstrip Detectors
}

\author{
RD 39 Collaboration
}

R. Wedenig and T.O. Niinikoski

CERN, Geneva, Switzerland

P. Berglund and J. Kyynäräinen ${ }^{\mathrm{a}}$

Helsinki University of Technology, Low Temperature Laboratory and Institute of

Particle Physics Technology, Otaniemi, Finland

L. Costa and M. Valtonen

Helsinki University of Technology, Department of Electrical and Communications Engineering, Otaniemi, Finland

R. Linna, J. Salmi, H. Seppä and I. Suni

VTT, Technical Research Centre of Finland, Otaniemi

\begin{abstract}
Summary
Superconducting $\mathrm{NbN}$ strip transmission line counters and coupling circuits were processed on silicon wafers using thin film techniques, and they were characterized with several methods to verify the design principles. The stripline circuits, designed using microwave design rules, were simulated using a circuit design tool enhanced to include modelling of the superconducting lines. The strips, etched out of the $282 \mathrm{~nm}$ thick top $\mathrm{NbN}$ film with resistivity $284 \mu \Omega \mathrm{cm}$ at 20 $\mathrm{K}$, have critical temperatures in the range 12 to $13 \mathrm{~K}$ and a critical current density approximately $J_{c}(0)=3.3 \cdot 10^{5} \mathrm{~A} / \mathrm{cm}^{2}$. The linearized heat transfer coefficient between the strip and the substrate is approximately $1.1 \cdot 10^{5} \mathrm{~W} /\left(\mathrm{m}^{2} \mathrm{~K}\right)$ and the healing length is about $1.6 \mu \mathrm{m}$ between 3 and $5 \mathrm{~K}$ temperatures. Traversing $5 \mathrm{MeV} \alpha$-particles caused the strips to quench. No events due to electrons could be detected in agreement with the predicted signal amplitude which is below the noise threshold of our wideband circuitry. The strip bias current and hence the signal amplitude were limited due to a microbridge at the isolator step of the impedance transformer.
\end{abstract}

(Submitted to Nuclear Instruments and Methods)

a Now at VTT, Technical Research Centre of Finland, Otaniemi 


\section{INTRODUCTION}

First tests [1] of a superconducting $\mathrm{NbN}$ strip detector, using near-minimum ionising electrons from a ${ }^{106} \mathrm{Ru}$ source, show good evidence for $5 \mathrm{mV}$ self-recovering signals with less than 300 ps rise time. The analysis $[1,2,3]$ of the results of these tests suggests that nonequilibrium phenomena could be responsible for the speed and self-recovery of the observed signals. A new regime of operation becomes therefore available, enabling one to obtain large signals in strips made of materials with high resistivity in the normal state.

The proposed $\mathrm{NbN}$ strip devices feature radiation hardness two orders of magnitude higher than conventional silicon strip detectors, spatial resolution limited only by lithographic techniques (typically $0.1-2 \mu \mathrm{m}$ ), an intrinsic signal rise time of $2 \mathrm{ps}$, and signal transport over large distances without losses. Comparing with the silicon strip and gas microstrip detectors, the Superconducting MicroStrip Detectors (SMSD) offer further advantages in the following areas

- Dead time

- Low-power signal processing and readout

- Mass per detector plane

It is generally thought that the study of physics processes requiring high-resolution vertex detection, in particular the detection of secondary vertices from $b-, t$ - and $\tau$-decays, is limited to the initial phase of LHC operation with low luminosities. The technology which we propose to investigate has the potential of overcoming this limitation.

The present initial study aims at improved understanding of the physics of such detectors, with the main goal of establishing their large-scale feasibility in applications such as the inmost vertex detectors of LHC experiments operated at high luminosities. The devices could have applications also in trackers for primary beams and for intense secondary beams.

In a vertex detector a relatively high field is required, and we have therefore chosen to study strip detectors made of the type II material $\mathrm{NbN}$ with high critical field, critical current density and critical temperature. Other high-resistivity materials such as PbBi alloys, Chevrel phase compounds, A15 compounds (such as $\mathrm{Nb}_{3} \mathrm{Ge}$ ), or HTSC perovskite materials are also attractive, but their study is beyond the scope of the present work. We report here the design and fabrication of $\mathrm{NbN}$ thin film microstrip circuits on silicon substrate, with long microstrip lines, and describe the characteristics of the $\mathrm{NbN}$ films and the strip lines at helium temperatures. These were obtained by measuring the critical current and temperature, normalstate resistivity, and the quench propagation parameters. Tracking detector tests were precluded by a processing problem which resulted in a weak link in the strips at the step of the isolator. Signals could therefore be detected with alpha particles only, and no self-recovering pulses could be observed.

\section{PRINCIPLE OF OPERATION}

Several schemes for implementing superconducting strip counters have been proposed and studied. At present one of them shows good promise and offers perspectives for vertex detectors and other applications where present technology limits the performance of the 
apparatus. In this scheme a portion of the superconducting microstrip line is driven normal by the heat generated by the passage of a particle through it. Such strips, made of a metal or a metallic compound, were suggested [4] for use at the SSC.

Two main models explain the behaviour of the transient phenomena leading to the detection of particles: 1) Equilibrium models, and 2) Non-equilibrium models. These differ in the extent of thermal equilibrium between the phonon and electronic systems of the material. A further class of models also takes into account the inertia of the conduction electrons, which leads to the concept of kinetic inductance, visible in very small lines.

\subsection{Equilibrium models}

The use of superconducting strips as an alpha particle detector was proposed already in 1962 by Sherman [5], and was demonstrated using Sn and In film strips in 1965 by Spiel, Boom and Crittenden [6]. The operation of the detector was shown to be bolometric; i.e. the bulk of the film material was heated up sufficiently by $\alpha$-particles so that a section underwent a transition to normal state. In this mode the pulse rise time may be limited by the speed of heat diffusion, which depends on the speed of phonons and on the electron-phonon scattering. From a thermodynamic model, Crittenden and Spiel [7] estimate the intrinsic pulse rise time $t_{r}=100$ ps.

The use of pure metal films has the inconvenience that the strip critical current and resistance in the normal state are low, both of which reduce the signal substantially. A systematic experimental study by Crittenden and Spiel [7] confirms that the diameter of the effective normal zone created by alpha particles is given by thermodynamic treatment and reads

$$
r_{c}=\sqrt{\frac{d E / d x}{\pi \Delta h}}
$$

where $\mathrm{d} E / \mathrm{d} x$ is the energy loss per unit thickness and $\Delta h$ the enthalpy difference per unit volume for the superconducting transition, given by

$$
\Delta h=\frac{\Delta H}{V}=\frac{B_{c t h 0}^{2}}{2 \mu_{0}} .
$$

Here the thermodynamic critical field at zero temperature $B_{c t h 0}$ can be calculated from the upper critical field $B_{c 2}$ and the Ginzburg-Landau parameter $\kappa$. A sputtered $\mathrm{NbN}$ film with $T_{c}=14 \mathrm{~K}$ and $\rho=130 \mu \Omega \mathrm{cm}$, the upper critical field is $B_{c 2}=16.3 \mathrm{~T}$ and $\kappa=52$; therefore $\Delta H / V=$ $1.2 \cdot 10^{11} \mathrm{MeV} / \mathrm{cm}^{3}[1]$.

Wagner and Gray [4] estimate that minimum ionising particles could produce a normal zone with radius $r_{\mathrm{c}}$ of a few hundred nm, which results in a normal zone of length $2 r_{\mathrm{c}}$ and a voltage drop

$$
V=2 r_{c} \rho J
$$

where $\rho$ is the resistivity in the normal state and $J \leq J_{\mathrm{c}}$ is the current density, slightly below the critical current density $J_{\mathrm{c}}$ of the material. In high-resistivity materials, pulses up to hundred $\mathrm{mV}$ could be expected, as opposed to (relatively) pure metals in where the pulse height tends to stay below one $\mathrm{mV}$. As an example, $\mathrm{NbN}$ may have a resistivity [8] above $200 \mu \Omega \mathrm{cm}$ and critical current up to $10^{7} \mathrm{~A} / \mathrm{cm}^{2}$ [9]. With $200 \mathrm{~nm}$ normal zone the resulting voltage pulse is $80 \mathrm{mV}$ 
using the above numbers. Adjusting the resistance of the normal zone by proper choice of the isolating film height so that impedance match with the line is near perfect, the signal observed at the end of the strip can be expected to have the same height and very nearly the same shape, because attenuation and dispersion are very low in superconducting transmission lines. These points will be described below in more detail.

If the strip switches to the normal state over the whole width in the region of the initial hot spot, one will see an expanding normal zone in case of a positive propagation speed of the hot zone limit. The minimum bias current $I_{\min }$ at which this happens is the current for zero propagation speed of a normal zone of length $x_{0}$; this is given by [10]

$$
I_{\text {min }}\left(x_{0}\right)=\sqrt{\frac{w^{2} h \alpha^{\prime}\left(T_{c}-T_{a}\right)}{\rho}} \cdot \sqrt{1+\sqrt{\frac{\kappa_{S}}{\kappa_{N}}} \operatorname{coth}\left(\frac{x_{0}}{\eta_{N}}\right) \operatorname{coth}\left(\frac{L / 2-x_{0}}{\eta_{S}}\right)},
$$

with $x_{0}=2 r_{0}, w$ and $h$ the width and thickness of the strip, $T_{a}$ the temperature of the substrate, $\rho$ the resistivity of the strip, $\kappa_{S}$ and $\kappa_{N}$ the thermal conductivities of the superconducting and normal regions of the strip, $\eta_{S}$ and $\eta_{N}$ the corresponding healing lengths, and $L$ the length of the strip. The constant $\alpha^{\prime}$ is related to the Kapitza conductivity $\alpha$ by $\alpha^{\prime}=4 \alpha T_{c}^{3}$ and is approximately $10^{5} \mathrm{~W} /\left(\mathrm{m}^{2} \mathrm{~K}\right)$ [1], and $T_{c}$ is the self-field critical temperature of the strip. The relation of Eq. (4) was derived under the assumption that the heat flux at the interface between the superconducting film and the substrate is linearly proportional to their temperature difference $\Delta T$, which holds only for small $\Delta T / T_{c}$. In our case this does not hold, but the error is acceptable in view of the fairly rough estimates of the remaining parameters. The parameters which can be extracted from the data are therefore accurate to about $\pm 50 \%$ only.

The thermal healing length $\eta$ as defined in Ref. [10] is given by

$$
\eta=\sqrt{\frac{\kappa h}{\alpha^{\prime}}}
$$

where $\kappa$ is the thermal conductivity of $\mathrm{NbN}$ in the normal state at $14 \mathrm{~K}$. We estimate $\kappa$ to be approximately $1 \mathrm{Wm}^{-1} \mathrm{~K}^{-1}$. The thermal conductivity in the superconducting state is close to this value also because the electronic contribution to it is relatively small at low temperatures. The resistivity $\rho$ of our $\mathrm{NbN}$ layer was measured to be $284 \mu \Omega \mathrm{cm}$ at $20 \mathrm{~K}$. With these numeric values the thermal healing length is of the order of $1.5 \mu \mathrm{m}$ for our strip thickness $h=$ $282 \mathrm{~nm}$.

\subsection{Non-equilibrium models}

Non-equilibrium phenomena play a decisive role in the initial evolution of the hot spot created by a fast traversing particle in a thin superconducting microstrip counter. The thermalization of the hot spot is a relatively slow process, and a rigorous treatment of the initial evolution problem would require the solution of the Boltzmann equation. This is clearly very difficult, although many simple cases can be rather well approximated by the Rothwarf-Taylor equations [11] which explain successfully the quasiparticle and phonon dynamics in tunnel junctions for example.

In addition to the quasiparticles and phonons, the electromagnetic fields need to be taken into account. This requires the simultaneous solution of Maxwell's equations. Superconductivity 
can be taken into account by adapting the Landau-Ginzburg equations into the time-evolution equations. We shall discuss these phenomena below mainly qualitatively and, where possible, semiquantitatively. In our discussion we shall refer to Fig. 1 which schematically illustrates the dynamics of the hotspot growth in a $\mathrm{NbN}$ film with $\rho_{N}=130 \mu \Omega \mathrm{cm}$ resistivity and $J=10^{6}$ $\mathrm{A} / \mathrm{cm}^{2}$ current density.

Relativistic particles traverse a $300 \mathrm{~nm}$ thick film in $1 \mathrm{fs}$. A major portion of the ionization deposited in the material is dissipated in the conduction electrons in about $10 \mathrm{fs}$. During this time quasiparticles, with Fermi velocity of $2.1 \mu \mathrm{m} / \mathrm{ps}$, may transport the heat to a distance of 20 $\mathrm{nm}$, if they are not scattered. The atomic oscillations have minimum periods of about $100 \mathrm{fs}$; in the $10 \mathrm{fs}$ time scale we may ignore the ionic lattice contribution to the sharing and transport of energy. The phonon system will begin to be heated by energetic electrons in about $1 \mathrm{ps}$ after the passage of the particle, but for thermal excitations near gap energy, the electron-phonon relaxation time is much longer, of order $\tau_{e-p h} \approx 60 \mathrm{ps}$ in $\mathrm{NbN}$ for example. The electronphonon relaxation process has been discussed recently by Gray [12].

Because quasiparticles will be ultimately scattered in the material, the propagation of the initial 'normal current zone' is controllable by the microscopic structure of the material, which may reveal itself very important in the present application.

Let us now assume that in the subpicosecond time scale, energy is mainly shared and transported by quasiparticles, and that the current with uniform density will continue to flow in the normal conducting volume, thus heating the quasiparticles and therefore enlarging the spot radius. This is depicted in Fig. 1a, which shows the hotspot with maximum diameter of $60 \mathrm{~nm}$ at approximate time $t=100 \mathrm{fs}$, sufficient for the propagation of the quasiparticles within the spot but clearly insufficient for sharing a substantial part of the energy with the equilibrium phonons.

The assumption of an almost undisturbed current distribution (Fig. 1a) may be justified in the subpicosecond time scale, because eddy currents will allow changes in the self-induced flux, and therefore in the current distribution, only very slowly. We estimate that in $\mathrm{NbN}$ the time constant for magnetic flux to creep out of the hotspot is of the order

$$
\frac{B}{\dot{B}} \cong \frac{B^{2}}{2 \mu_{0} \rho_{N} J^{2}} \cong \frac{\mu_{0} d^{2}}{2 \rho_{N}} \cong 0.5 \mathrm{ps}
$$

In the zone heated above the critical temperature, once greater in diameter than the correlation length $(\approx 5 \mathrm{~nm})$, the current flow will therefore be dissipative, and a voltage drop will occur in it. An electric field will also leak out of the film, if the hotspot traverses the film, as is expected in the case of fast projectiles. The electric field pulse will begin to propagate in the dielectric at a speed characteristic of the superconducting line.

In the first picoseconds the energy dissipated in the electronic system by the bias current cannot be coupled to the phonons, and one may make the simple assumption that this energy will heat and enlarge the non-equilibrium 'quasiparticle hotspot'. We estimate the growth speed of the radius in the following simple way. Equating the Joule heat generated in the hot spot of area $A$, with the rates of energy change due to the growth of the spot and due to the coupling of the electrons with the phonons, one obtains the equation 


$$
\rho_{N} J^{2} A d=\Delta h \dot{A} d-\frac{C_{e}\left(T_{c}-T_{b}\right)}{\tau_{e-p h}} A d .
$$

Here $T_{c}$ is the critical temperature, $T_{b}$ is the phonon temperature (close to the initial substrate temperature), and $d$ is the strip thickness. Because of the long correlation time $\tau_{e-p h}$ between the electrons and phonons in $\mathrm{NbN}$, the last term is small compared with the first one on the right side, and we can get a simple expression for the growth time constant of the surface of the hot spot:

$$
\tau_{A}=\frac{\Delta h}{\rho_{N} J^{2}} \approx 0.7 \mathrm{ps}
$$

in the case of $\mathrm{NbN}$. Assuming that the spot is circular and will not deform in the very beginning, the radius will the grow exponentially with an initial rate of

$$
\dot{r}=\frac{r}{2 \tau_{A}} \approx 40 \mathrm{~nm} / \mathrm{ps}
$$

so that the initial "equilibrium" radius of $60 \mathrm{~nm}$, due to a MIP, would be roughly doubled in about 1 ps. The current and flux, however, move out of the centre of the spot somewhat faster as shown by Eq. (6), enabling the quasiparticles of the currentless centre of the spot to cool at a speed determined by the quasiparticle heat transfer to the surface of the strip ( $\approx 100 \mathrm{fs})$, by the phonon-quasiparticle correlation time $(\approx 60 \mathrm{ps})$, by the acoustic mismatch of the phonons at the film-substrate interface, and, after phonons are heated in the whole volume, by the time for an acoustic phonon to traverse the film $\tau_{p h}=d / v_{a} \approx 100 \mathrm{ps}$. The hot spot now becomes a hot ring, heated on the perimeter by the current but cooled in the centre by heat transfer between the electronic and phonon systems, and via the interface between the film and the substrate. The ring diameter at $t=1 \mathrm{ps}$ (Fig. 1b) is of the order of the thermal healing length in $\mathrm{NbN}$, and it has become slightly oval because the heated ring-shaped zone will expand faster towards the sides of the strip than along its length, due to the fact that the current density will grow on the sides whereas it will drop on the ends of the ring.

In the next picosecond the ovalized ring will grow very fast in the direction perpendicular to the original current direction, until it will touch one of the sides of the strip (Fig. 1c). The speed may approach the Fermi velocity $v_{F} \approx 2 \mu \mathrm{m} / \mathrm{ps}$. The whole few-micrometer width of a narrow strip may thus be bridged by a dissipative non-equilibrium hot zone in less than 2 picoseconds, because the current density on the sides of the ovalized ring may exceed the intrinsic critical current of the material.

It is rather logical to expect a rapid growth of the hotspot in the case of a high-resistivity film carrying a high current density. It is much more difficult to explain how the hot zone may recover in such a case; intuition says that nothing might prevent the axial extension of the zone, and in the framework of the equilibrium model this is supported by the calculated minimum current beyond which the hot spot will expand [10]. However, our non-equilibrium model, which was qualitatively described above, gives two possible mechanisms providing selfrecovery of the hotspot: Firstly, the current distribution across the strip is highly non-uniform just after the whole width of the strip has been bridged by the hot zone, and secondly the quasiparticles may have time to condense back into the superconducting ground state in the currentless areas, due to electron-phonon heat transfer. This may be occurring first in the 
proximity of the film-substrate interface, which is also strongly suggested by the results showing a substantial dependence of the pulse characteristics on the substrate quality [7].

The reason for the non-uniform current distribution across the strip in the hot zone is schematically illustrated in Fig. 1c. Because usually one or the other side of the film is reached first by the hot ring, current density there may drop to zero as soon as the self-induced magnetic flux may rearrange itself. In the high-resistivity material this will be much more rapid than in the pure metals; granular films may also be better in this sense. The final heated zone will certainly have a length in the direction of the strip which is highly non-even across the strip. The current density will therefore readjust itself in a way which reflects the shape of the zone; the readjustment, however, does not happen instantaneously but within the rough time scale of Eq. (6). During this time a supercurrent may start flowing in those areas which carry no or low normal current; the dynamics of this supercurrent zone will now determine whether the original state of the strip can be self-recovered. The dynamics of a supercurrent zone is different from that of a closed zone with normal current, because the electromagnetic field behaves in a different way in these two areas, and because the relative directions of the gradients of various quasi-thermodynamic quantities (heat, magnetic flux, $\partial J / \partial t$ etc.) will be changed in these two cases. The growth of the supercurrent zone is expected to be much slower, and it may depend heavily on the geometry of the hot zone.

It may be actually required that the third dimension should be included in the quantitative estimates of the hot zone recovery, because when the film is contacted only on interface with the substrate, large vertical gradients can appear already during the growth of the nonequilibrium hot zone. This is particularly plausible when the hot zone length is of the same order of magnitude as the height of the film, as was the case in $\mathrm{NbN}$ [1]. The supercurrent "shortcircuit" can thus appear on the interface in an area of low normal current density; the supercurrent density in such thin layer may have a very much higher intrinsic critical limit due to the restricted geometry of the layer.

The formation of the self-recovering electromagnetic pulse in the stripline is explained qualitatively as follows: Let us imagine a spot with normal current, giving an electric field in the material due to Ohm's law. The field leaks out above and below the film, and actually fieldlines also reach the return conductor which partly shorts the hot zone. The resulting timedependent electric field, appearing in the isolator between the stripline and the return ground plane, is associated with displacement current and resulting magnetic field; the electromagnetic pulse will start to propagate first radially as a planar guided wave, and after reaching the sides of the strip, it will propagate along the strip in opposite directions with opposite polarities. One of the waves will reach the shorted end of the line, and it will be reflected back with opposite polarity so that it will combine with the first wave constructively. An observer (amplifier) in the other end of the line will see first the half-pulse propagating towards it, and after a time delay required for the propagation of the opposite pulse back and forth between the hot zone and the shorted end of the line, he will see a superposed step of equal height, making up the voltage drop in the resistive zone. There is thus no restriction from superconducting currents shorting the normal one, and the first edge of the signal is associated with the first dissipative hotspot rather than with the normal zone bridging the strip. The initial pulse rise time is therefore expected to be faster than the 2 ps time estimated for the expansion of the hot ring until the sides of the strip. 
If the downstream end of the strip is open instead of short circuited (allowing, however, for the dc bias current to flow) for the high-frequency pulse, the reflected wave will not have opposite polarity. The two waves will therefore combine destructively, and the observer will see a pulse with amplitude one-half of the voltage across the normal spot, and duration equal to the time delay required for the propagation of the opposite pulse back and forth between the hot zone and the open end of the line. These timing features might be useful for extracting the information on the location of the hotspot along the strip.

\section{SUPERCONDUCTING TRANSMISSION LINES}

Because an important potential advantage of superconducting microstrip line counters is the combination of fast pulses with slow propagation along the line, making serial readout possible, we shall briefly review here the theory of the superconducting microstrip transmission line.

\subsection{Speed of signal propagation}

The speed of signal propagation (phase velocity) in a normal strip line is approximately

$$
v=\frac{c}{\sqrt{\varepsilon_{r, e f f}}} .
$$

If the conductors are in the superconducting state, the signal propagation is slower than this because the magnetic field can penetrate about $\lambda \sim 50-500 \mathrm{~nm}$ into the metal, while electric fields are almost completely excluded [13]. This results in the signal propagation speed $[14,15]$ which is constant at frequencies below the gap frequency and is given approximately by

$$
v=\frac{c}{\sqrt{\varepsilon_{r, e f f}}} \frac{1}{\sqrt{1+2 \frac{\lambda}{h} \operatorname{coth} \frac{d}{\lambda}}} .
$$

For thin isolators ( $h \sim 1-2 \mathrm{~nm}$ in Josephson junctions) this can be less than one percent of the speed of light, and can be of order of few percent of $c$ in the case of $20 \mathrm{~nm} \mathrm{TiO}_{2}$ dielectric; taking $w=2 \mu \mathrm{m}, h=20 \mathrm{~nm}, d=300 \mathrm{~nm}, \lambda=100 \mathrm{~nm}$ and $\varepsilon_{r, e f f}=80$, we find $v=0.0338 c$. If the superconducting films are also thinner than the penetration depth, the propagation speed is reduced even further. This effect is due to the kinetic energy of the charge carrier electrons, which can be included in the field equations as an additional inductance, called kinetic inductance. The properties of the superconducting transmission lines can thus be estimated by replacing the normal inductance of the line by the sum of the magnetic and kinetic inductances.

The theoretical attenuation [15] in superconducting microstrip lines is due to losses in the dielectric and in the superconductors:

$$
\begin{gathered}
\alpha_{d}=\frac{\omega}{2 v} \tan \delta \\
\alpha_{s}=\frac{\omega^{2} \lambda^{3} \mu_{0} \varepsilon_{r, e f f} \sigma v}{2 h c^{2}}\left[\operatorname{coth} \frac{d}{\lambda}+\frac{d}{\lambda} \sinh ^{-2} \frac{d}{\lambda}\right],
\end{gathered}
$$

where $\tan \delta$ is the dielectric loss tangent and $\sigma$ is the conductivity of the charge carriers in the normal state 


$$
\sigma=\sigma_{N}\left(\frac{T}{T_{c}}\right)^{4} .
$$

The resulting attenuation in $\mathrm{NbN}$ microstrip lines is very small, and using the dimensions of our planned detector the above equations yield the attenuation less than $10^{-2} \mathrm{~dB} / \mathrm{m}$ at frequencies below the gap frequency, provided that the operating temperature is below $0.2 T_{\mathrm{c}}$. Such low attenuation and the resulting very low dispersion has been beautifully demonstrated using sub-picosecond pulses and optical sampling techniques [16, 17].

We conclude that the detector strip length can be several metres, and that signal transport far from the central region is also possible without degrading the signal quality and timing resolution, provided that the operating temperature is stable.

\subsection{Impedance matching}

The impedance of a normal strip line is given by

$$
Z_{L}=Z_{0} \frac{h}{w_{\text {eff }}} \sqrt{\frac{1}{\varepsilon_{r, e f f}}},
$$

where $Z_{0}=\sqrt{\mu_{0} / \varepsilon_{0}} \approx 120 \pi \Omega, h$ is the thickness of the isolating film on the ground plane and $\varepsilon_{\mathrm{r}, \text { eff }}$ its effective dielectric coefficient at the frequency of operation. The effective width $w_{\text {eff }}$ is calculated from an empirical formula

$$
\frac{w_{\text {eff }}}{h}=\frac{w}{h}+2.46-0.49 \frac{h}{w}+\left(1-\frac{h}{w}\right)^{6},
$$

where $w$ is the geometric width of the strip. In practice the width of the strip is always much larger than the dielectric height in our case, and therefore the fringing field correction is at most a few percent.

In superconducting materials the penetration of electric and magnetic fields in the conductor behaves in a different manner, and the above formulas cannot be directly applied in the case of isolator thickness $h \sim 20 \mathrm{~nm}$. Kautz [18] has analysed such lines in terms of the theory of Mattis and Bardeen [19] for the complex conductivity of a BCS superconductor. At frequencies below the gap frequency $(\sim 1 \mathrm{THz})$ the impedance is constant and can be approximated by

$$
Z_{S C}=Z_{0} \frac{h}{w_{\text {eff }}} \frac{1}{\sqrt{\varepsilon_{r, e f f}}} \sqrt{1+\frac{2 \lambda}{h} \operatorname{coth} \frac{d}{\lambda}},
$$

where $\lambda \sim 50-500 \mathrm{~nm}$ is the magnetic field penetration depth in the superconducting material. This equation also takes into account the kinetic inductance of the microstrip line.

If $w=2 \mu \mathrm{m}, h=20 \mathrm{~nm}, d=300 \mathrm{~nm}, \lambda=100 \mathrm{~nm}$ and $\varepsilon_{r, \text { eff }}=80$, we find $Z_{L}=0.41 \Omega$ and $Z_{S C}=1.35 \Omega$. Such low impedance will need to be matched to normal transmission lines when using normal electronics for detector testing. We propose to study the possibility of using a stripline impedance transformer to perform the matching, and we plan to integrate thin film impedance transformers with the detector, in order to match the microstrip impedance around $1 \Omega$ with the standard $50 \Omega$ impedance of coaxial lines, thus conserving the widest possible bandwidth in the course of tests. 
The impedance matching of the superconducting transmission lines may be necessary not only if normal $50 \mathrm{ohm}$ transmission lines and electronics will be used, but also for matching the strips with possible superconducting logic circuits.

\subsection{Stripline components, Josephson junctions and other superconducting circuits}

In addition to the matching circuits required for adopting the signal strips with the transmission lines and readout, also other stripline components could be built on the detector substrate. The potentially useful circuits include at least capacitors, inductors, directional couplers, splitters, combiners, loads, shorts etc. The motivation for such functions on the substrate is mainly in the possibility of analogue handling of the signals before entering into the superconducting logic, with view on possibly simplifying the readout logic.

The advantages of Josephson logic are not only ultra-high speed and remarkable compactness, but also extremely low power consumption. Operating in the microwatt range, the Josephson logic would provide almost negligible heat load in the interior of the detector, whereas this is a major problem with conventional semiconductor logic. It is well known, however, that HEMT devices and also CMOS circuits work at helium temperatures, although their design is not optimised for such low temperatures.

The first practical realisation of Josephson logic functions was developed by IBM [20]. The current injection logic (CIL) [21] used several Josephson junctions for each logic function, and is capable of reasonable fan-in and fan-out so that large logic arrays could be easily designed. The single logic levels were not clocked but latched. The total IBM gate delays were 27 to $45 \mathrm{ps}$ for OR and AND functions, with power dissipations 1.5 to $6.0 \mu \mathrm{W}$. Data are read at initial latch and propagate asynchronously through the logic, and are again synchronised at output latches. This suits very well for operation in the readout logic of a collider detector, where the latch frequency can be the accelerating frequency or its harmonic. The vertex detector could thus be easily read out during the $25 \mathrm{~ns}$ between the LHC bunch crossings, and it might be possible to resolve events separated by less than one nanosecond. Clock frequencies of 10-30 GHz are possible.

The first superconducting logic circuits suffered from the fabrication technology which was based on soft and oxidizing materials such as lead alloys. The advent of devices based on niobium metal and $\mathrm{Al}_{2} \mathrm{O}_{3}$ isolator films, and in particular the development of the Rapid Single Flux Quantum (RSFQ) logic family [22] has led to commercial fabrication of practical devices such as fast ADC's, signal correlators and TDC's. The RSFQ logic gates contain a flip-flop and can use clock speeds which are theoretically much higher than the CIL logic which needs resetting by the latching pulses.

The standard Josephson gates cannot be operated at high fields, which is a handicap for vertex detectors. It could, however, be envisaged that in many applications the readout logic could be sufficiently shielded from the magnetic field by miniature superconducting shields and/or compensation coils, and they could be located to a region of low field and low flux of particles. 


\section{PREPARATION OF THE STRIPLINE CIRCUITS AND THE TEST EQUIPMENT}

\subsection{Circuit and mask design}

The aims of the circuit design were defined as follows:

1) Design microstrip lines as narrow as possible to maximize the spatial resolution of the detector

2) Achieve sufficiently low impedance level to maximize the energy of the pulse

3) Achieve slow propagation speed to optimize the spatial resolution along the line

4) Design a broadband impedance circuit to match the impedance of the microstrip line to the $50 \Omega$ line and to maximize the signal power reaching a pulse analyzer

5) Inject the DC bias current into the microstrip line without disturbing the pulse propagation

6) Design the transition to the AC contact pads without introducing discontinuity in the transmission line impedance

7) Design AC pads so that contact to the $50 \Omega$ coaxial transmission line can be made without significant microwave reflection.

8) Design a set of the test circuits which can be used to measure the DC characteristics of the lines and, in addition, to measure the propagation speed and attenuation constant of the transmission line.

The model for the microstrip line used in the design is based on the inductance equations derived specially for the superconducting microstrip lines. The equations also include fringing field effects so that narrow lines can also be accurately analyzed. The capacitance of the superconducting microstrip line is identical to that of the normal conducting line. The thicknesses of the line, of the ground plane, and of the insulation layer are such that the London penetration depth (about $300 \mathrm{~nm}$ ) has a marked influence both on the impedance level and the on propagation speed. The inductance of the double stripline was assumed to be twice that of the superconducting microstrip line. The impedance of the other lines was estimated based on the inductance of the microstrip line and the double stripline. The equation for the pulse height generated by the kinetic inductance (due to the reduction of the number of the Cooper pairs) was developed and was used to optimize the parameters of the detector.

We were unsure concerning the physics behind the pulse generation. The model based on the kinetic inductance were used in the design only because it enabled us to find out, at least, a rough estimation for the pulse height. The first mask design was made without using the models implemented in the circuit simulation and design tool APLAC [23].

To meet all the requirements, the following ideas in the mask and circuit design were used:

1. The microstrip line is divided into two parts with different thicknesses of the insulating layer: for the actual detector it is $25 \mathrm{~nm}(\mathrm{aSi}: \mathrm{H})$ and for the line entering the contact pads it is $300 \mathrm{~nm}\left(\mathrm{SiO}_{2}\right.$.) The amorphous hydrogenated silicon was selected because it was used in high frequency microstrip lines by several groups.

2. The width of the line in the detector area is $2 \mu \mathrm{m}$.

3. The thickness of the line on the step formed by the two insulation layers is more than 20 nm. 
4. The ground plane under the contact pads forms a narrow microstrip line $(2 \mu \mathrm{m})$ in order to maintain the $50 \mathrm{Ohm}$ impedance level in the contact pads.

5. The thickness of the ground plane is about $100 \mathrm{~nm}$ in order to get an appropriate inductance.

6. The thickness of the microstrip line is about $300 \mathrm{~nm}$ to keep the critical current as high as possible but still maximize the pulse height related to the kinetic inductance.

7. A tapered microstrip line is used for the impedance matching circuit. If the impedance of the transmission line increases slowly compared to the wavelength of the incident wave, no marked reflection occurs. For this reason we prefer the tapered line to the transformer based on lumped elements.

The complete design of the circuit is shown in Fig. 2. In the central part of the chip there are four active detector striplines of different lengths, each meander-shaped line covering a rectangular surface. The tapered impedance matching circuits - the transformers - are located on the sides. The rounded pads are the AC contacts and the rectangular ones provide DC current feed and voltage sense connections to AC pads via inductive strips patterned on the circuit in the same process.

Due to the wide frequency bandwidth the tapered lines requires plenty of room. The transformer can also be completed by using lumped elements leading to a more compact design. In the future when the characteristics of the elements will be predictable, lumped elements will be used instead of the tapered lines, to maximize the detector area.

\subsection{Simulation of superconducting circuits}

A model for a superconducting microstrip line was implemented [24] in the circuit simulator and design tool APLAC [23]. The model included the frequency-dependent losses in the dielectric in terms of the frequency dependence of the effective permittivity of the microstrip, so accounting for dispersion. The impedance transformer was modelled and simulation indicated good characteristics up to $10 \mathrm{GHz}$ and beyond as is shown in Fig. 3. A pulse arising from a change in the kinetic inductance of the active part of the stripline was also simulated and studied.

\subsection{Processing of the first series of chips}

Before processing a series of tests were carried out in order to optimize the parameters of each processing step. Deposition of the $\mathrm{NbN}$ superconductive films were carried out in a vacuum chamber, evacuated using an oil diffusion pump, using reactive DC magnetron sputtering from a $\mathrm{Nb}$ target. First the current-voltage characteristics of the sputter gun was measured with different argon/nitrogen sputtering gas mixtures. A batch of $\mathrm{NbN}$ films were then deposited using different $\mathrm{Ar} / \mathrm{N}_{2}$ ratios, gas total pressures, and substrate temperatures. Constant sputtering voltages were used to give stable deposition just above the transition between the $\mathrm{Nb}$ and $\mathrm{NbN}$ type of deposition. The films were characterized by measuring the RT resistivity, the resistance ratio between RT and $20 \mathrm{~K}$, the critical temperature, and the critical current density at $4.2 \mathrm{~K}$. The stoichiometry of the films were measured using ion backscattering. The $\mathrm{Nb}$ to $\mathrm{N}$ ratio of the films varied between 0.89 and 1.40 , and all films were found to contain oxygen. The critical current densities varied between 1.6 and $3.5 \cdot 10^{6} \mathrm{~A} / \mathrm{cm}^{2}$, 
and the critical temperature up to $13.8 \mathrm{~K}$ was monitored. For the first microstrip detectors the sputtering parameters of a film deposited at $425{ }^{\circ} \mathrm{C}$ were chosen which gave an RT resistivity of $207 \mu \Omega \mathrm{cm}$, resistance ratio of 0.73 , critical temperature of $13.0 \mathrm{~K}$, and critical current density of $3.5 \cdot 10^{6} \mathrm{~A} / \mathrm{cm}^{2}$ in a film of $85 \mathrm{~nm}$ thickness and $\mathrm{Nb}: \mathrm{N}: \mathrm{O}$ atom composition of $1: 0.97$ : 0.16 .

The deposition of the amorphous hydrogenated silicon, a:Si-H, was made in the same system using RF sputtering from a $\mathrm{Si}$ target in $70 / 30 \mathrm{Ar} / \mathrm{H}_{2}$ gas. High total pressures were found to be necessary to decrease the Ar content in the films. Films deposited at RT were easily wet etchable, but leaky at RT. Deposition at $425{ }^{\circ} \mathrm{C}$ produced leak free films which could not be etched selectively on $\mathrm{NbN}$ with wet chemicals.

To process the first detectors a ground layer $\mathrm{NbN}$ was first sputtered and patterned with Reactive Ion Etching (RIE) with $\mathrm{SF}_{6}$ gas. The a:Si-H first dielectric layer was then deposited and patterned either using $\mathrm{SF}_{6}$ RIE (RD-01) or wet chemical etching with TMAH (RD-02). In the latter case a separate $\mathrm{SiO}_{2}$ masking layer deposited with Plasma Enhanced Chemical Vapour Deposition (PECVD) was used. The $\mathrm{SiO}_{2}$ second dielectric layer was then deposited with PECVD and patterned with wet etching using buffered HF. The stripline layer $\mathrm{NbN}$ was deposited and patterned in a similar manner compared to the ground plane. Finally the resistive Pd layer was e-gun evaporated and patterned with a liftoff method. The thicknesses of the five layers are given in Table 1 below.

\begin{tabular}{|lrr|}
\hline Layer & RD-01 & RD-02 \\
\hline \hline NbN ground layer & $102 \mathrm{~nm}$ & $102 \mathrm{~nm}$ \\
a:Si-H (ellipsometer measurement) & $25 \mathrm{~nm}$ & $25 \mathrm{~nm}$ \\
$\mathrm{SiO}_{2}$ & $300 \mathrm{~nm}$ & $300 \mathrm{~nm}$ \\
$\mathrm{NbN}$ strip layer & $282 \mathrm{~nm}$ & $282 \mathrm{~nm}$ \\
$\mathrm{Pd}$ & $251 \mathrm{~nm}$ & $271 \mathrm{~nm}$ \\
\hline
\end{tabular}

Table 1: Thicknesses of the five films of the RD-01 and RD-02 wafers of RD39.

\subsection{Test equipment}

Two cryostats were been built for characterising the thin-film samples and for testing the processed chips. One of the cryostats, shown in Fig. 4, features continuous flow operation with a base temperature of $1.75 \mathrm{~K}$ and cooldown in about 1 hour. The cryostat has thin windows for beam access or for electrons from a $106 \mathrm{Ru}$ source, and instrumentation for thermometry and the control of temperature and flow. The sample chips were initially mechanically contacted and required mounting and contact adjustment under microscope. An improved PCB chip carrier with wire bonded contacts was used in the later phases of the studies to be described below.

The heat sinking of the coaxial and DC lines was also improved and special cryogenic microcoaxial lines [25] were fabricated and mounted in the cryostat.

The data acquisition and control system for the measurements of the critical currents was set up at CERN. The apparatus is based on a multifunction DAQ board in a Nubus slot of a Macintosh microcomputer running LabVIEW programs for the various tasks. The voltage drop over the strip during the I-V characterisation was limited by a series of two diodes connected in 
parallel with the strip under study. The temperature was measured using a calibrated germanium thermometer and silicon diode sensors using a multichannel self-balancing bridge circuit [26].

The equipment of the transmission line characterization at CERN consists of a Tektronix Time Domain Reflectometer (TDR) in a 7104 mainframe oscilloscope equipped with a CCD camera for capturing the video images of the oscilloscope screen into a PC. Tektronix software is used for converting the video files into numeric transient records. These, in turn, are converted by LabVIEW into ASCII files for use in the data analysis in spreadsheet and graphics programs.

The pulse observation equipment consists of wideband linear amplifiers and a $1 \mathrm{GHz}$ vertical amplifier unit in the Tektronix mainframe. The CCD camera can capture the video image of the signal at the fastest writing speed of $10 \mathrm{~cm} / \mathrm{ns}$ of the oscilloscope. Faster features can be only indirectly studied by measuring the shot noise due to the particle interactions, using a microwave spectrum analyzer.

\section{RESULTS OF THE FIRST SET OF DETECTOR CHIPS}

\subsection{Measurements of the critical current and the critical temperature}

The self-field critical current was determined from the measured the I-V characteristic of the strip as a point where the voltage exceeded a certain threshold value. The measurement was performed using a 4-point technique which was made possible by the design of the mask patterns. The sample chips were initially mechanically contacted and required mounting and contact adjustment under microscope. An improved PCB chip carrier with wire bonded contacts was used in the studies be described below and the results are compared with the old ones.

The strips showed three different behaviours. In Figure 5a we can see an example for the simplest case. Up to the critical current $I_{\mathrm{c}}$ the resistant remains zero, and at $I=I_{\mathrm{c}}$ a sudden voltage rise occurs, which indicates the phase transition to the normal conducting state. Many of the strips of batch 1 show this behaviour. A more complicated pattern is also observed where there is a first step to a resistive behaviour, before the whole strip quenches.

In Figure $5 b$ an I-V scan is shown for a strip which exhibits a pronounced 3-step behaviour. In all cases the positions of the steps were rather reproducible and the lowest step was the most reproducible one. We interpret the two- and three-step features as a characteristic of the microbridges which may form at both ends of the strip, where there is a step of the isolating film in the junction between the stripline and the impedance transformer. Figure 6 shows the end of the detector stripline meander and its junction with the impedance transformer.

The appearance of steps in the I-V curve can be explained by the fact that the heating produced by the current passing through the microbridge in the normal state may be below the value at which the propagation velocity of the normal zone in the narrow strip is positive and leads to a stable situation in which the slope of the I-V curve gives the resistance of the microbridge. Typical values of the resistance were $100-500 \Omega$. However, if the resistance of the microbridge is high enough, the whole strip may quench immediately, and therefore it is possible to observe two, one or no steps before quench.

Some I-V scans are shown in Fig. 7 at different temperatures, for a mechanically contacted strip which exhibited a pronounced 2-step behaviour. In such a case the position of the lower 
step was always more reproducible and was considered as the self-field maximum current of the weakest point of the strip; this does not describe the behaviour of the stripline itself.

The critical temperature $T_{\mathrm{c}}$ and the maximum current at absolute zero $I_{C}(0)$ were determined by a fit of the temperature dependence of the maximum current of each strip to the law

$$
\frac{I_{c}(T)}{I_{c}(0)}=1-\left(\frac{T}{T_{c}}\right)^{2}
$$

Some fits are shown in Figure 8 and Figures 9a,b.

The measured strips, wire bonded (Figure 8) or mechanically mounted (Figure 9), featured critical temperatures around $12 \mathrm{~K}$, in agreement with earlier results of VTT on unprocessed test films. The low-temperature critical currents were $2.5 \mathrm{~mA}$ at best, indicating critical current densities in the range $3 \cdot 10^{5} \mathrm{~A} / \mathrm{cm}^{2}$ if the transition occurs in the strip with nominal width. This current density is lower than the best results elsewhere by a factor of 30-100, and about 10 times lower than the test films discussed above. We believe that this discrepancy is due to a microbridge which is formed at the junction to the impedance transformer where a poor coverage of the step in the isolator thickness may occur, if the step is steep and the growth of the $\mathrm{NbN}$ film takes place vertically only.

Some strips showed that superconductivity persisted at low current values at least $1 \mathrm{~K}$ above the $T_{c}$ determined from the fit of the lower temperature data. Such a tail is clearly visible in Fig. $9 \mathrm{~b}$ ), and is often interpreted as a sign of fine granularity with pronounced grain boundaries which limit the value of the critical current density.

\subsection{Circuit studies using a complex network analyser}

The contact resistance between the coaxial line and the contact pads were found to be near $50 \Omega$. The attenuation of the microstrip line was measured using a network analyzer from 1 $\mathrm{MHz}$ to $6 \mathrm{GHz}$. The temperature of the substrate was not accurately known during the measurements. However, in the experiments where the temperature of the chip was below the transition temperature of the $\mathrm{NbN}$ line and ground plane, an attenuation higher than $10 \mathrm{~dB}$ was observed. The measured attenuation included the reflection due to the contact resistance and the attenuation generated by the coaxial cable between the analyzer and sample holder. The result implies either that the silicon insulation layer generates dissipation or the flux is creeping in the superconducting film leading to the AC dissipation. Since the attenuation was observed to reduce markedly as the temperature was lowered well below the transition temperature, we believe that the flux creep is responsible for the observed high attenuation. Although the dissipation of the insulation layer depends slightly on temperature, it is not very likely that the small drop in temperature implies such a large change in attenuation.

\subsection{Circuit studies using a time domain reflectometer (TDR)}

The TDR images feature a clear but unexpected change at the critical temperature of the strip material. A sequence of scans are shown in Fig. 10 which lead to the conclusion that the coaxial line is dominantly capacitively shunted at temperatures above $T_{c}$ and becomes resistively terminated with large resistance when the films become superconducting. The likely explanation of the observations is that the ground strip in the impedance transformer quenches into the normal state because the TDR pulse current is $4 \mathrm{~mA}$ and the thickness of the ground film is 
around $100 \mathrm{~nm}$. With equal critical current densities in the ground and upper $\mathrm{NbN}$ films, the ground film cannot support the supercurrent of the size of the pulse current in any section under the contact pad, in the impedance transformer, nor in the strip.

The TDR pulse current was lowered by inserting a $20 \mathrm{~dB}$ attenuator in the coaxial line between the instrument and the cryostat. The reflected signals remained qualitatively similar to those with the full $200 \mathrm{mV}$ pulse amplitude.

One of the strips, however, showed clearly a successful impedance transform in one end, which indicates that the design principles are correct.

\subsection{Pulses due to charged particles}

The chips were exposed to radioactive ${ }^{241} \mathrm{Am},{ }^{90} \mathrm{Sr}$ and ${ }^{106} \mathrm{Ru}$ sources of $10 \mathrm{kBq}, 3.6 \mathrm{MBq}$, $150 \mathrm{kBq}$ intensities, respectively. Figure 11 shows a typical signal due to an alpha particle. All the pulses were very well correlated with quenches and have a rise time of about $250 \mathrm{~ns}$. Unfortunately the pulse rate was very low. This made it difficult to distinguish pulses due to thermal fluctuations from those due to the traversing particles, because no trigger detector was available.

To clarify this we quantified the temperature dependence of the critical current, both with and without source. Since $\alpha$-particles deposit more energy than electrons, they were most likely to show clear results. Data taken with and without source is shown Figure 12. The I-T scans agree very well, but unfortunately there are only few measurements points which indicate an event. The two points at $3.5 \mathrm{~K}$ and $5.1 \mathrm{~K}$ show definitely lower values of the quench current. They are interpreted to be due to the energy deposit of an $\alpha$-particle traversing the $\mathrm{NbN}$ film, creating a hot spot which leads to the quench of the stripline at a bias current below the critical current of the microbridge.

The expected quench rate based on the size of the hot spot and the particle rate can be calculated using the energy loss obtained by taking the derivative of the empirical range [27]

$$
\ln \left(\frac{d}{1 \mu \mathrm{m}}\right)=B_{1}+B_{2} \ln E+B_{3}(\ln E)^{2}+B_{4}(\ln E)^{3}+B_{5}(\ln E)^{4}+B_{6}(\ln E)^{5}
$$

where $A_{\mathrm{i}}$ are the coefficients for the projected range given in Table 2 for $\mathrm{Nb}$ and $\mathrm{N}$, and $E$ is the initial energy of the particle in $\mathrm{keV}$. The ${ }^{241} \mathrm{Am}$ source features peaks of the alpha spectrum at $5.388 \mathrm{MeV}, 5.443 \mathrm{MeV}$ and $5.486 \mathrm{MeV}$, of which the last dominates with a transition probability of $85.2 \%$. Inserting the energy of the dominant peak into Eq. (19) gives a penetration depth $d$ of $12.076 \mu \mathrm{m}$ in $\mathrm{Nb}$. The energy loss rate is $d E / d x=3.11 \mathrm{GeV} / \mathrm{cm}$ in a pure $\mathrm{Nb}$, whereas $d E / d x=0.93 \cdot 10^{-3} \mathrm{GeV} / \mathrm{cm}$ in gaseous $\mathrm{N}_{2}$, for the average alpha particle energy of $E=5.41 \mathrm{MeV}$.

\begin{tabular}{|lcccccc|}
\hline & $B_{1}$ & $B_{2}$ & $B_{3}$ & $B_{4}$ & $B_{5}$ & $B_{6}$ \\
\hline \hline $\mathrm{Nb}$ & -5.2876 & 0.75572 & 0.16023 & -0.045036 & 0.0040297 & $-8.4665 \mathrm{e}-5$ \\
$\mathrm{~N}$ & -5.9816 & 1.0111 & 0.02475 & -0.026042 & 0.0028908 & $-5.2713 \mathrm{e}-5$ \\
\hline
\end{tabular}

Table 2: Coefficients to calculate projected ranges of $\alpha$-particles in $\mathrm{Nb}$ and $\mathrm{N}_{2}$.

The energy loss rate in $\mathrm{NbN}$ is obtained by taking the weighted sum of the loss rates of the constituents 


$$
\frac{d E_{\mathrm{NbN}}}{d x}=\frac{w_{\mathrm{Nb}} \rho_{\mathrm{NbN}}}{\rho_{\mathrm{Nb}}} \frac{d E_{\mathrm{Nb}}}{d x}+\frac{w_{\mathrm{N}} \rho_{\mathrm{NbN}}}{\rho_{\mathrm{N}}} \frac{d E_{\mathrm{N}}}{d x}=3.47 \mathrm{GeV} / \mathrm{cm}
$$

where

$$
w_{i}=\frac{a_{i} A_{i}}{\sum_{i} a_{i} A_{i}}
$$

and $a_{i}$ is the molar fraction of element $i$ with atomic weight $A_{i}$. With the densities $\rho_{\mathrm{NbN}}=8.47 \mathrm{~g} / \mathrm{cm}^{3}, \rho_{\mathrm{Nb}}=8.60 \mathrm{~g} / \mathrm{cm}^{3}$ and $\rho_{\mathrm{N}}=0.0013 \mathrm{~g} / \mathrm{cm}^{3}$, Eq. (1) yields the radius of the initial hot spot $r_{0}=0.96 \mu \mathrm{m}$ in $\mathrm{NbN}$ using Eq. (20). In $\mathrm{Nb}$ film an experimental value of $1.2 \mu \mathrm{m}$ [28] for $\alpha$-particle induced hot spot radius has been determined using a different method based on Abrikosov vertex density in a Josephson tunnel junction. This agrees rather well with our estimate based on the upper critical field value, in view of the lower heat capacity of $\mathrm{Nb}$ compared with $\mathrm{NbN}$.

To estimate the expected quench rate we assume a point source at the distance $R=10 \mathrm{~mm}$ from the strip area $a b$ and calculate the particle rate $\dot{N}_{s}$ through the strip with the filling factor $L w / a b$

$$
\dot{N}_{s} \cong \frac{\dot{N}_{t o t} a b}{4 \pi R^{2}}\left(\frac{L w}{a b}\right)=\frac{\dot{N}_{t o t} L w}{4 \pi R^{2}}=22 \mathrm{~s}^{-1}
$$

where $a=3 \mathrm{~mm}$ and $b=4 \mathrm{~mm}$ are the dimensions of the strip area, $L=70 \mathrm{~cm}, w=4 \mu \mathrm{m}$, and $\dot{N}_{\text {tot }}$ is the total number of disintegrations.

The I-V scan is done with a scan rate of $10 \mathrm{~mA} / \mathrm{s}$, so to reach a bias current of $2 \mathrm{~mA}$ it takes $\Delta t_{\text {scan }}=0.2 \mathrm{sec}$, which leads to a rate of about 4.4 particles per scan in the low temperature region. However, in the framework of the equilibrium model the strip is active for detecting the particles only when the current is higher than the threshold current $I_{t h}$

$$
I>I_{t h}=I_{c} \frac{w-2 r_{0}}{w}=0.52 I_{c},
$$

where $I_{c}$ is the self-field critical current of the detector strip. The expected average number of counts per scan $\dot{N}_{d} \Delta t_{\text {scan }}$ is then

$$
\dot{N}_{d} \Delta t_{s c a n}=\dot{N}_{s} \Delta t_{s c a n} \frac{I_{w p}-I_{t h}}{I_{w p}},
$$

where $I_{w p}$ is the limiting current at which the strip quenches because of the weak points at the ends of the strip.

From the data shown in Fig. 12 we estimate roughly $\dot{N}_{d} / \dot{N}_{s} \approx 2 / 13$ in the temperature range 2 to $6 \mathrm{~K}$ which yields

$$
\frac{I_{t h}}{I_{w p}}=1-\frac{\dot{N}_{d}}{\dot{N}_{s}} \cong 0.85
$$

This is consistent with the observed quench current ratios of 0.84 obtained directly from the data.

The internal consistency of the alpha particle-induced quench threshold current suggests that Eq. (22) can be used for determining the self-field critical current. The result for $T=0$ is 


$$
I_{c}(0)=0.85 \cdot I_{w p}(0) \frac{w}{w-2 r_{0}}=3.8 \mathrm{~mA}
$$

and gives the critical current density of $3.3 \cdot 10^{5} \mathrm{~A} / \mathrm{cm}^{2}$ which is by a factor of roughly 4 lower than the critical current densities measured with the films tested before the processing of the final batch of wafers. The discrepancy may be explainable in view of the differences of the geometry, film quality and the fact that the test films were in direct contact with the silicon substrate rather than the underlying $\mathrm{NbN}$ and isolator films.

The fact that the observed alpha-events were associated with a quench can now be used for determining iteratively the upper limit for the heat transfer coefficient and the lower limit for the healing length from the Eqs. (4) and (5). Using our parameters $w=4 \mu \mathrm{m}, h=282 \mathrm{~nm}$, $T_{c}=12 \mathrm{~K}, \kappa_{S}=\kappa_{N}=1 \mathrm{Wm}^{-1} \mathrm{~K}^{-1}$ and $L=70 \mathrm{~cm}$ we find $\alpha^{\prime}<1.2 \cdot 10^{5} \mathrm{~W} /\left(\mathrm{m}^{2} \mathrm{~K}\right)$ and $\eta>1.6 \mu \mathrm{m}$ at $T_{a}=3.5 \mathrm{~K}$, and $\alpha^{\prime}<1.0 \cdot 10^{5} \mathrm{~W} /\left(\mathrm{m}^{2} \mathrm{~K}\right)$ and $\eta>1.7 \mu \mathrm{m}$ at $5.1 \mathrm{~K}$. These agree very well with the value $\alpha^{\prime}=1.5 \cdot 10^{5} \mathrm{~W} /\left(\mathrm{m}^{2} \mathrm{~K}\right)$ found in [1] for $\mathrm{NbN}$ on sapphire.

\subsection{Self recovering pulses}

The expected self-recovering pulse height with $1.5 \mathrm{~mA}$ current in the $4 \mu \mathrm{m}$ strip with $284 \mu \Omega \mathrm{cm}$ normal resistivity is below $0.5 \mathrm{mV}$ with minimum-ionising particles. Such pulses are not detectable in our wideband system; we did not indeed succeed in observing any such pulses with electrons. The maximum currents in our strips are those of the step in the impedance transformer, and therefore the desired parameters could not be measured the in the current range above $5 \mathrm{~mA}$ which is of interest for the self-recovering pulses.

\section{CONCLUSIONS}

The $4 \mu \mathrm{m}$ wide strips etched out of the $282 \mathrm{~nm}$ thick top $\mathrm{NbN}$ film have critical temperatures in the range 12 to $13 \mathrm{~K}$ and a critical current density approximately $J_{c}(0)=3.3 \cdot 10^{5} \mathrm{~A} / \mathrm{cm}^{2}$. The linearized heat transfer coefficient between the strip and the substrate is approximately $1.1 \cdot 10^{5} \mathrm{~W} /\left(\mathrm{m}^{2} \mathrm{~K}\right)$ and the healing length is about $1.6 \mu \mathrm{m}$ between 3 and $5 \mathrm{~K}$ temperatures. The resistivity of the $\mathrm{NbN}$ of the top film is $284 \mu \Omega \mathrm{cm}$ at $20 \mathrm{~K}$ temperature.

Pulses due to $5 \mathrm{MeV} \alpha$-particles caused the strips to quench. No pulses due to electrons could be detected; they were presumably below the noise threshold of our wideband circuitry.

We conclude that the fabrication of multilayer structures involving two superconducting $\mathrm{NbN}$ planes with interleaved thin isolating film is feasible. Furthermore, we have shown that a step in the thickness of the isolating film is technically possible although it may lead to a critical microbridge. This step enables the transformation of the impedance of the line which, in turn, allows a larger amount of the available pulse energy to reach the readout circuitry. The processing of the upper film pattern requires a refinement so that the critical microbridge can be avoided.

The lower $\mathrm{NbN}$ film thickness of $102 \mathrm{~nm}$ leads to a critical current which is well below $1 \mathrm{~mA}$. This requires modifying the high-frequency test instruments and forces to operate them in a mode which is noisy. A thicker film could improve the situation. Alternatively one could avoid the impedance transformer, and design the readout circuitry so that the particle detection and tracking studies can be made with lower signals. 


\section{REFERENCES}

[1] B. Strehl, T. Niinikoski, A. Rijllart, K. Winter and M. Caria, Phys. Lett. B242 (1990) 285 .

[2] T.O. Niinikoski, A. Rijllart, B. Strehl, K. Winter and M. Caria, in Proc. 25th Int. Conf. on High Energy Physics, Ed. K. K. Phua and Y. Yamaguchi (World Scientific, Singapore 1991) p. 1349.

[3] T.O. Niinikoski, A. Rijllart, B. Strehl, K. Winter and M. Caria, in Proc. 12th Workshop of the INFN ELOISATRON Project, New Technologies for Supercolliders, Ed. L. Cifarelli and T. Ypsilantis (Plenum, New York 1991) p. 223.

[4] R.G. Wagner and K.E. Gray, in Proc. Int. Workshop on Superconductive Particle Detectors (World Scientific, Singapore 1988) p. 204.

[5] N.K. Sherman, Phys. Rev. Lett. 8 (1962) 438.

[6] D.E. Spiel, R.W. Boom and E.C. Crittenden, Appl. Phys. Lett. 7 (1965) 292.

[7] E.C. Crittenden and D.E. Spiel, J. Appl. Phys. 42 (1971) 3182.

[8] D.D. Bacon, A.T. English, S. Nakahara, F.G. Peters, H. Schreiber and W.R. Sinclair, J. Appl. Phys. 54 (1983) 6509.

[9] M.A. Janocko, J.R. Gavaler, C.K. Jones and R.D. Blaughter, J. Appl. Phys. 42 (1971) 182.

[10] W.J. Skocpol, M.R. Beasley and M. Tinkham, J. Appl. Phys. 45 (1974) 4054.

[11] A. Rothwarf and B.N. Taylor, Phys. Rev. Lett. 19 (1967) 27.

[12] K.E. Gray, in Proc. Int. Workshop on Superconductive Particle Detectors (World Scientific, Singapore 1988) p. 1.

[13] M. Tinkham, Introduction to Superconductivity (McGraw-Hill, New York 1975).

[14] J.C. Swihart, J. Appl. Phys. 32 (1961) 461.

[15] J.M. Pond, K.R. Carroll and E.J. Cukauskas, J. Appl. Phys. 27 (1991) 2696.

[16] W.J. Gallagher, C.-C. Chi, I.N. Duling, III, D. Grischkowsky, N.J. Halas, M.B. Ketchen and A.W. Kleinsasser, Appl. Phys. Lett. 50 (1987) 350.

[17] J. F. Whitaker and et al., Jap. J. Appl. Phys. 26 (1987) 1563.

[18] R.L. Kautz, J. Appl. Phys. 49 (1978) 308.

[19] D.C. Mattis and J. Bardeen, Phys. Rev. 111 (1958) 412.

[20] J. Matisoo, IBM J. Res. Develop. 24 (1980) 113.

[21] T.R. Gheewala, IBM J. Res. Develop. 24 (1980) 130.

[22] K.K. Likharev, Dynamics of Josephson junctions and circuits (Gordon and Breach, 1986).

[23] M. Valtonen, P. Heikkila, H. Jokinen and T. Veijola, APLAC - Object-Oriented Circuit Simulator and Design Tool, in Low-Power HF Microelectronics - a unified approach (IEE, London 1996).

[24] Luis J. Costa, Implementation of a Superconducting Microstrip Line Model, Helsinki University of Technology Master's Thesis (1995).

[25] R. Wedenig, Ch. Knoblauch and T.O. Niinikoski, Cryogenics 38 (1998) 239. 
[26] J. Ylöstalo, P. Berglund, T.O. Niinikoski and R. Voutilainen, Cryogenics 36 (1996) 1033

[27] J.F. Ziegler, Helium Stopping Powers and Rages in All Elements (Pergamon Press, 1977).

[28] R. Cristiano, L. Frunzio, S. Pagano and V.G. Palmieri, J. Appl. Phys. 82 (1997) 5024. 


\section{FIGURE CAPTIONS}

Figure 1: Dynamics of the non-equilibrium hot spot; see text for explanation.

Figure 2: Design of the layout of the thin-film circuit, showing the pattern of the top $\mathrm{NbN}$ film with four stripline meanders in the centre (the lines cannot be resolved in this scale), four impedance transformers on each sides, and four AC contact pads on the top and the bottom. The sides of the $20.20 \mathrm{~mm}^{2}$ chip are uncovered to make contact to the ground $\mathrm{NbN}$ plane shown in grey shade. The rectangular DC contact pads are connected to the AC pads via inductive lines which are invisible in this scale.

Figure 3: Elements of the complex scattering matrix $S$ of the superconducting taper designed to match the active strip to a $50 \Omega$ coaxial line. Shown are the magnitudes of $S_{11}$ (lowest curve) and $S_{21}$ (topmost curve), and the phase of $S_{11}$ (middle curve).

Figure 4: Assembly drawing of the RD39 test cryostat. The cryostat is mounted directly onto a LHe transport dewar and is fed by a straight vertical vacuum isolated transfer line (pointing left in the drawing) ending into a gas-liquid phase separator. The gas is used for cooling the two thermal radiation screens and the liquid is expanded via a cryogenic needle valve into an evaporator pot which is integrated with the mounting surface of the sample probing jig. Recessed windows allow sources and other detectors to be placed close to the detector chip under test.

Figure 5a: I-V characteristics of strip A of chip $3 / 3(w=4 \mu \mathrm{m}, L=70.47 \mathrm{~cm})$ at $2.5 \mathrm{~K}$.

Figure 5b: I-V characteristics of strip A of chip $2 / 3(w=4 \mu \mathrm{m}, L=39.67 \mathrm{~cm})$ at $7.5 \mathrm{~K}$.

Figure 6: Junction between the detector stripline and the impedance transformer.

Figure 7: Result of a series of five scans for a strip with a pronounced two-step feature in the I-V plot. This feature was not seen in all measured strips.

Figure 8: Temperature dependence of the maximum supercurrent of strip A of chip 3/3 $(w=4 \mu \mathrm{m}, L=70.47 \mathrm{~cm})$.

Figure 9: Self-field critical current as a function of the temperature of the copper frame of the sample probe, for a) strip A on chip RD-01/4/1 $(w=3 \mu \mathrm{m}, L=99.87 \mathrm{~cm})$ and b) strip A on chip RD-01/2/3 ( $w=3 \mu \mathrm{m}, L=150.97 \mathrm{~cm}$ ). The temperature difference between the chip and the copper frame was measured to be $1.0 \mathrm{~K}$ for $\mathrm{b}$ ) and was estimated to be slightly higher for a). The curves represent the fit of Eq. (18) to a) all data, b) data below $11.5 \mathrm{~K}$, and the insets show the parameters yielded by the fit.

Figure 10: Plots of Time Domain Reflectometer displays at different temperatures above and below the critical temperature of the $\mathrm{NbN}$ films of the strip A $(w=3 \mu \mathrm{m}, L=150.97$ $\mathrm{cm})$. The strip B $(w=3 \mu \mathrm{m}, L=39.67 \mathrm{~cm})$ on the same chip RD-01/2/3 has identical behaviour. The reflection coefficient is 0 at roughly 0.23 of the vertical scale. The first bump of the curves is the hermetic SMA connector at the entrance into the cryostat. The dotted lines which correspond to temperatures above $T_{c}$ feature a dip in the impedance at the entrance of the stripline, whereas solid lines which correspond to $T \approx 5 \mathrm{~K}$ indicate that the line is seemingly terminated with a high impedance at the contact pad.

Figure 11: Signal with rise time of $250 \mathrm{~ns}$ due to an $\alpha$-particle.

Figure 12: Temperature dependence of the maximum supercurrent of strip A of chip 3/3 $(w=4 \mu \mathrm{m}, L=70.47 \mathrm{~cm})$ with and without radioactive source. 

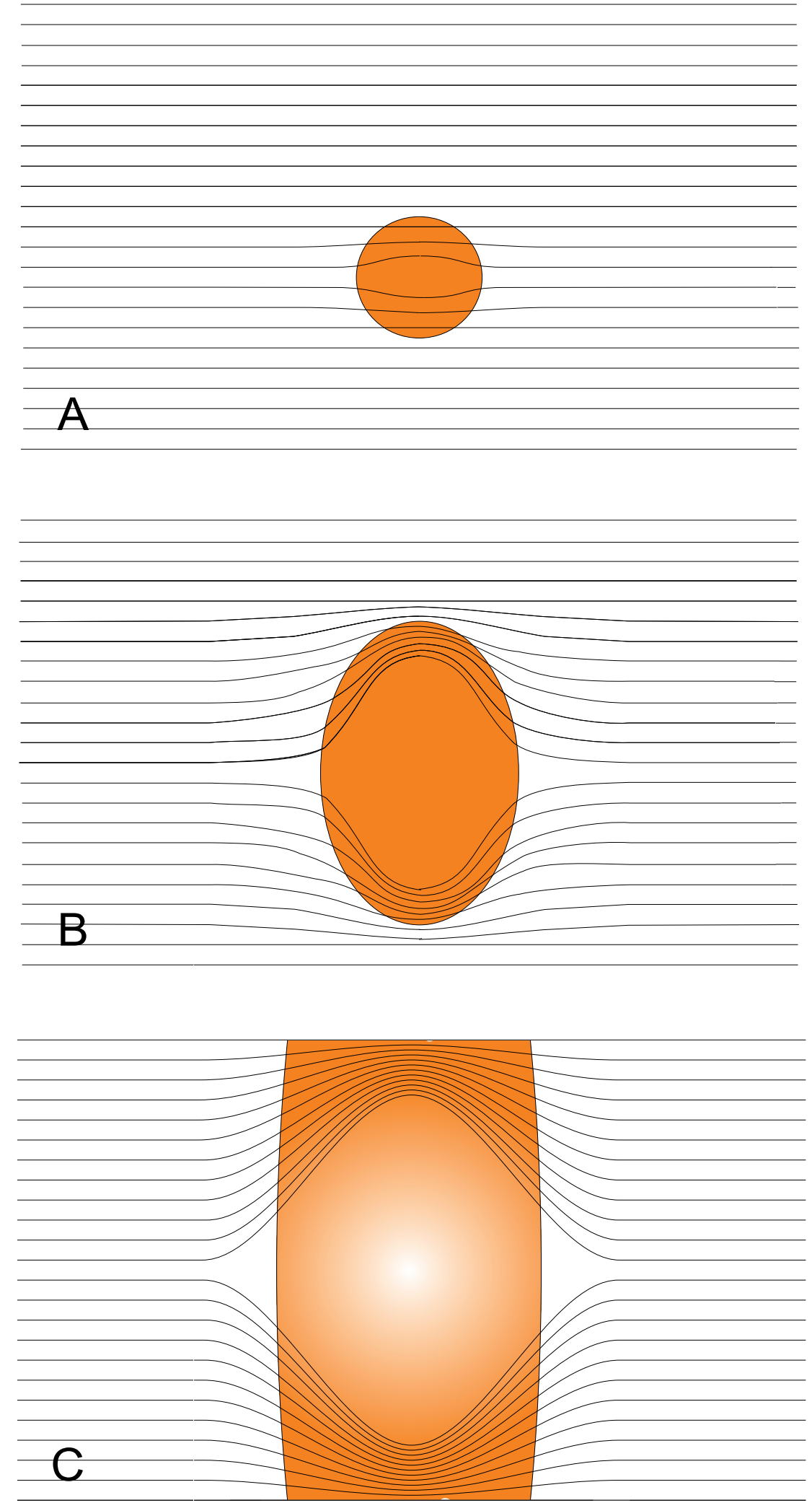

Figure 1 


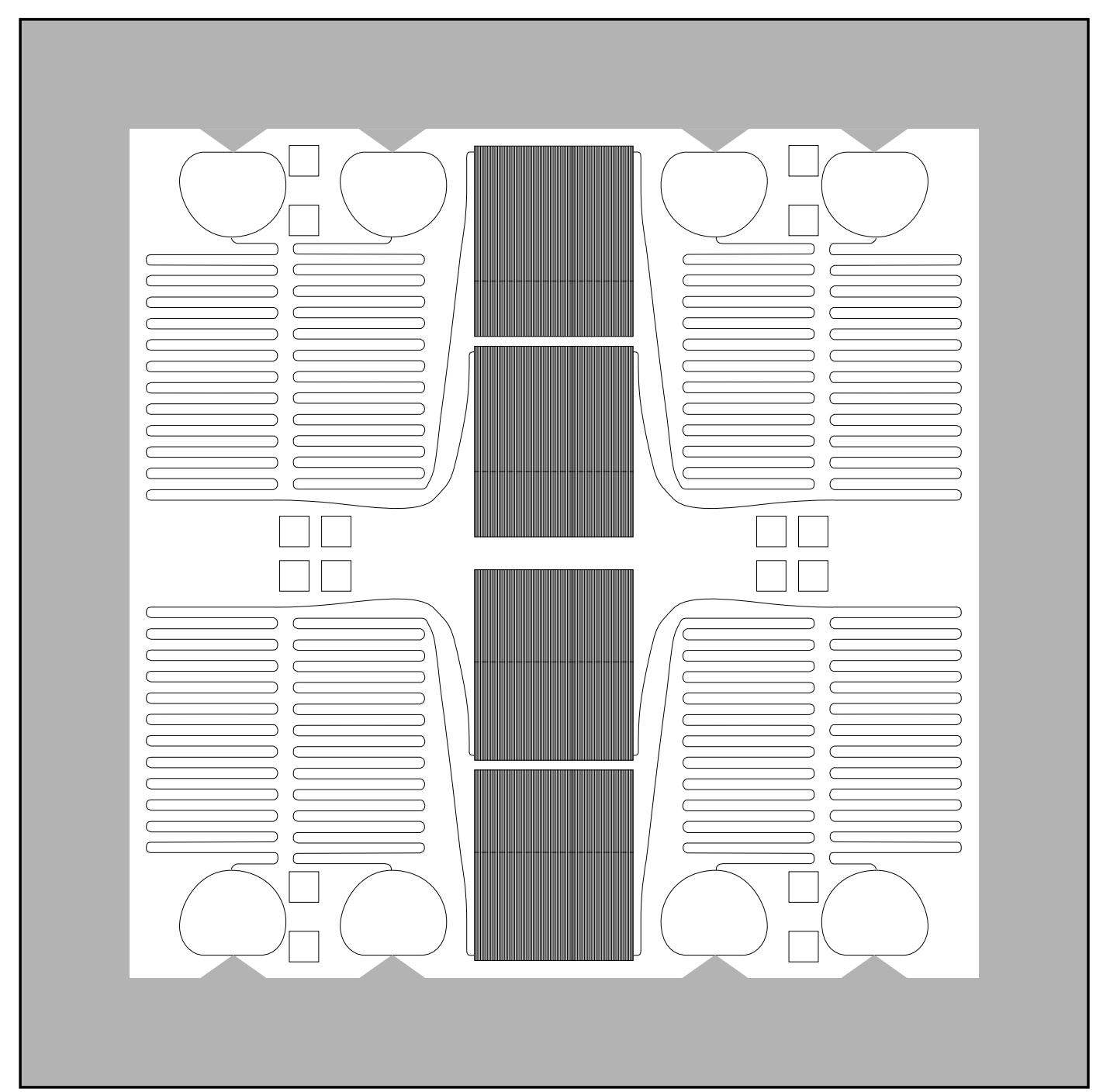

Figure 2

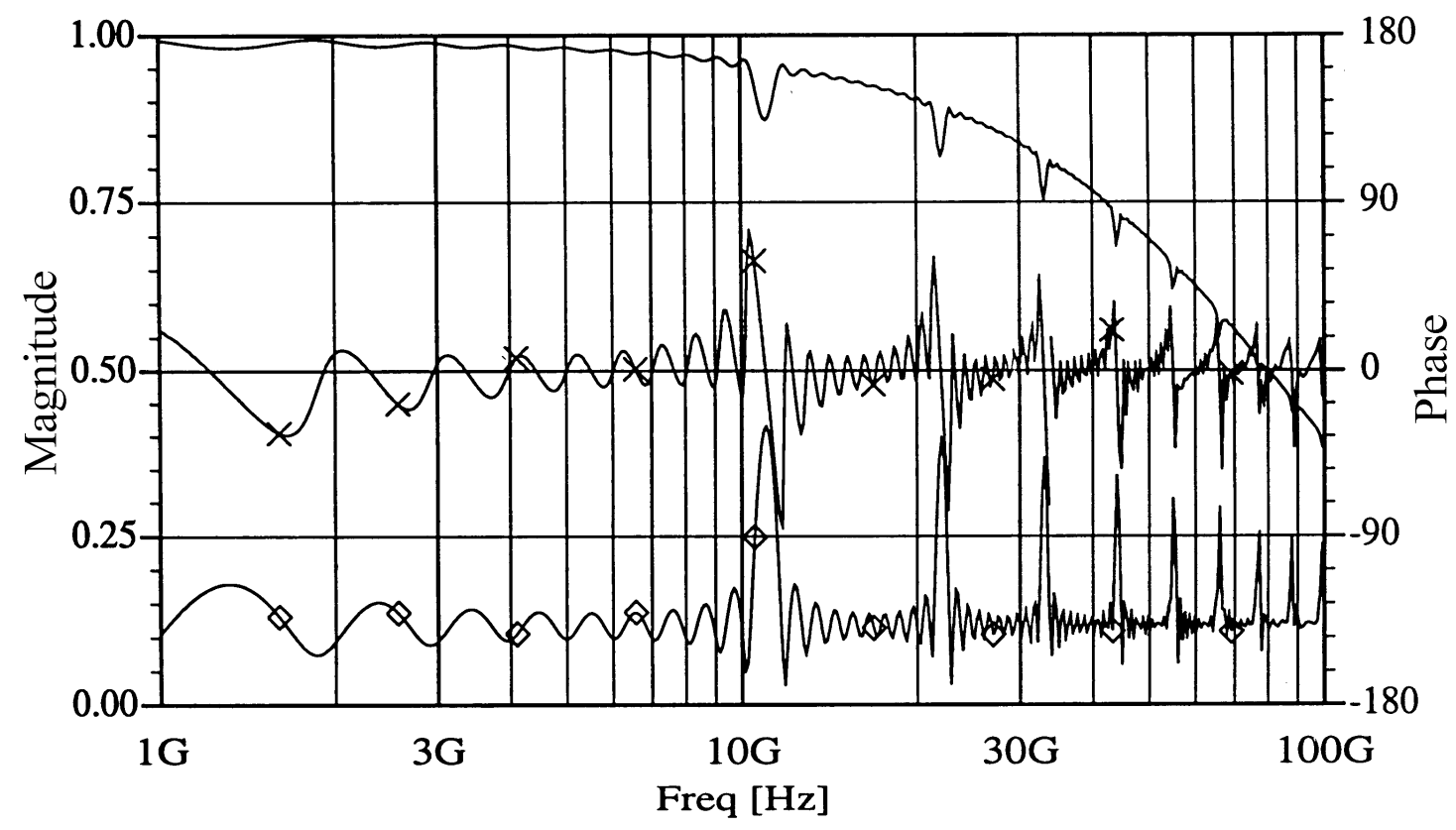

Figure 3 

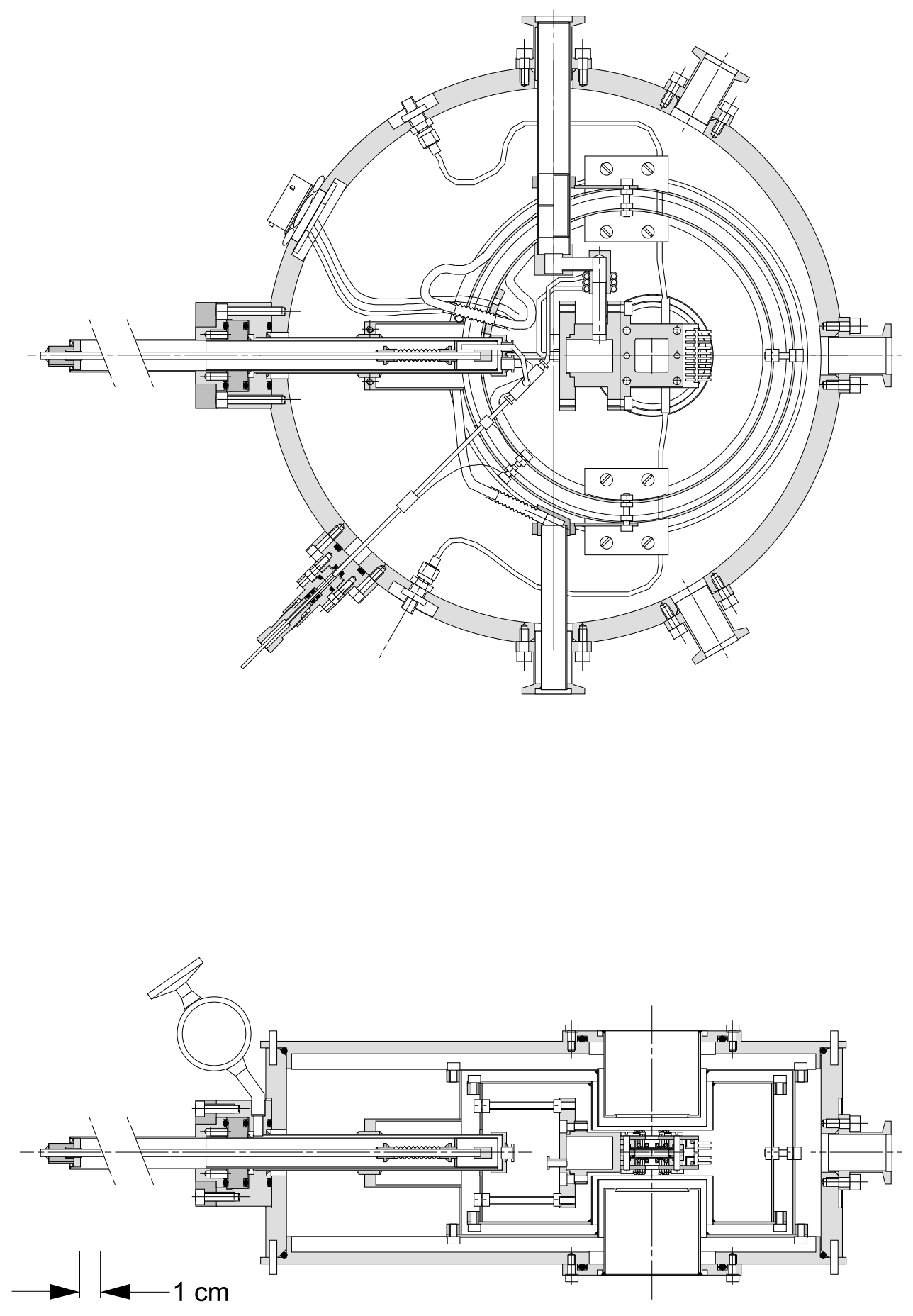

Figure 4 


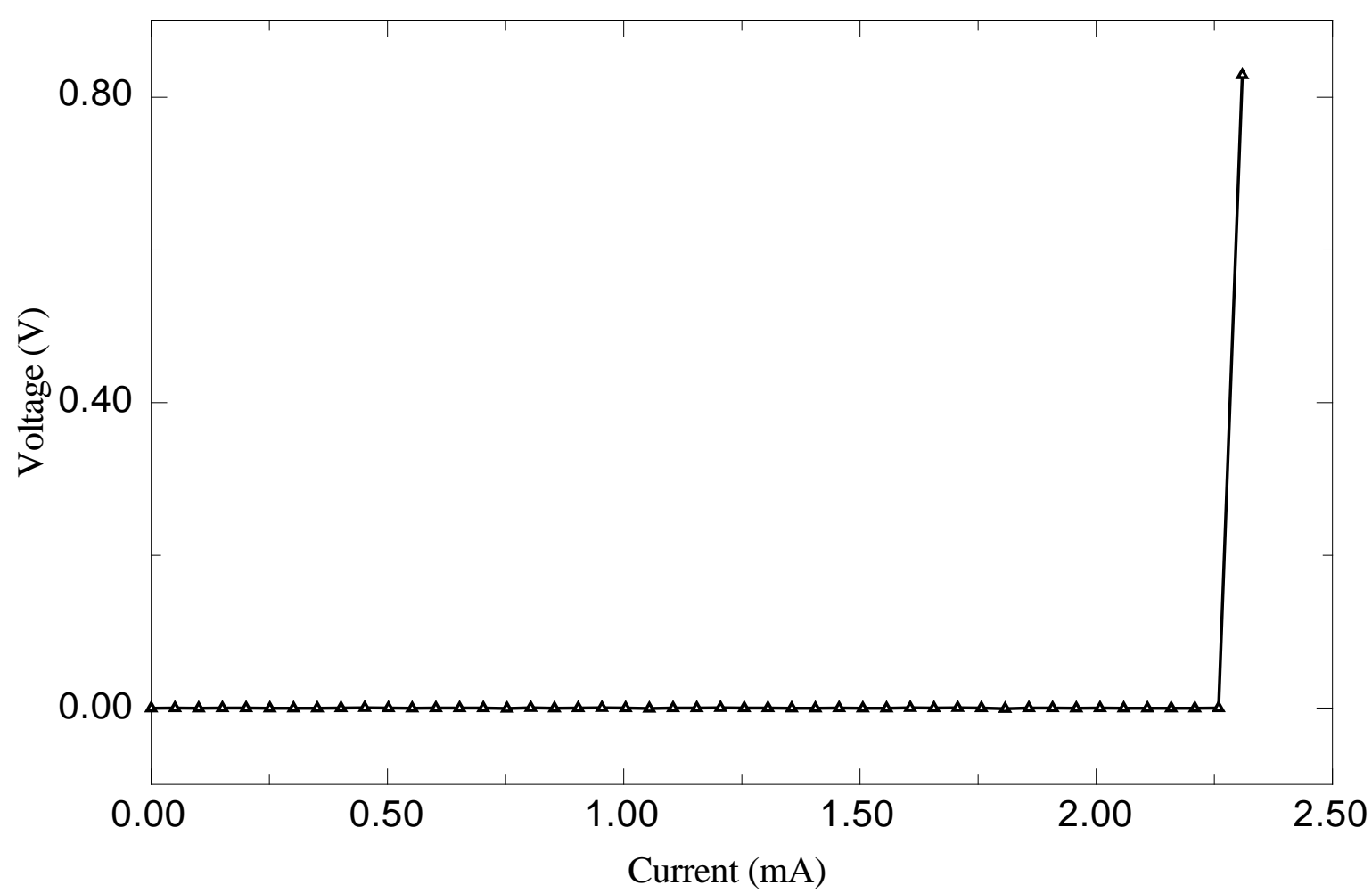

Figure 5a

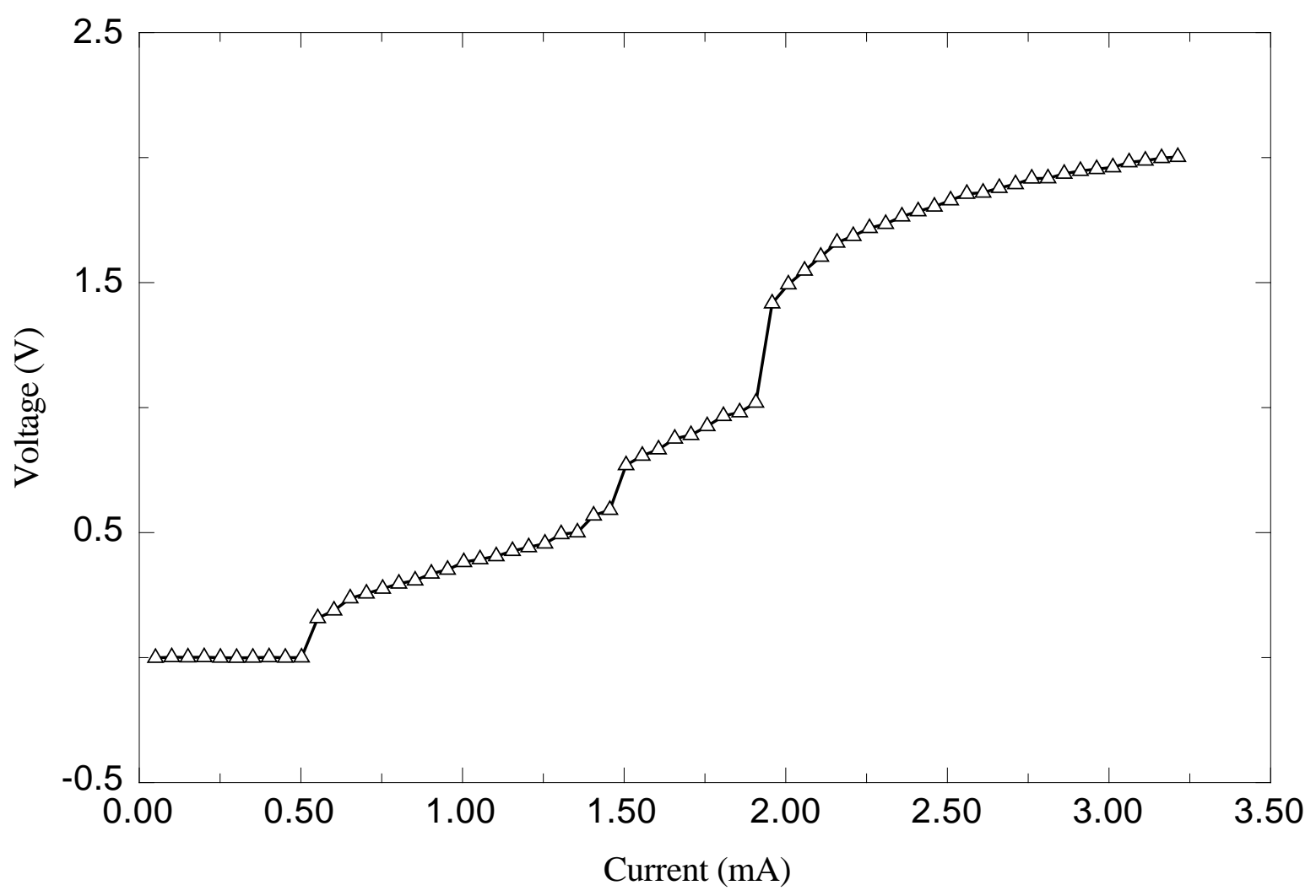

Figure $5 b$ 


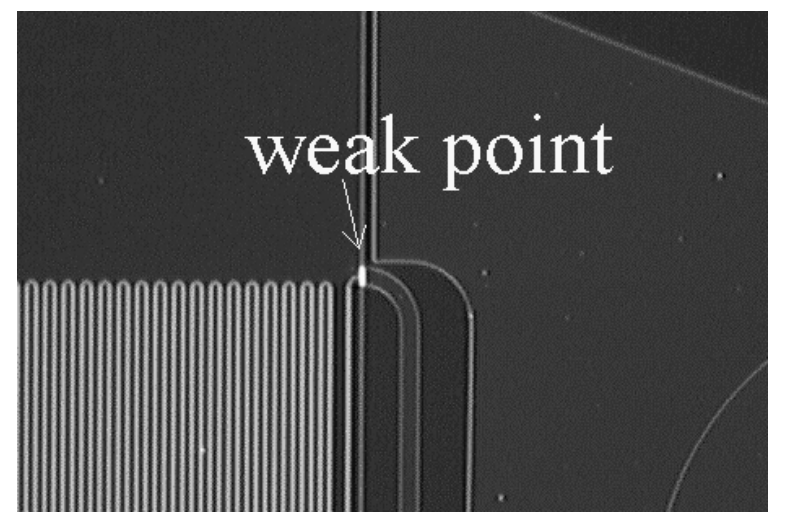

Figure 6

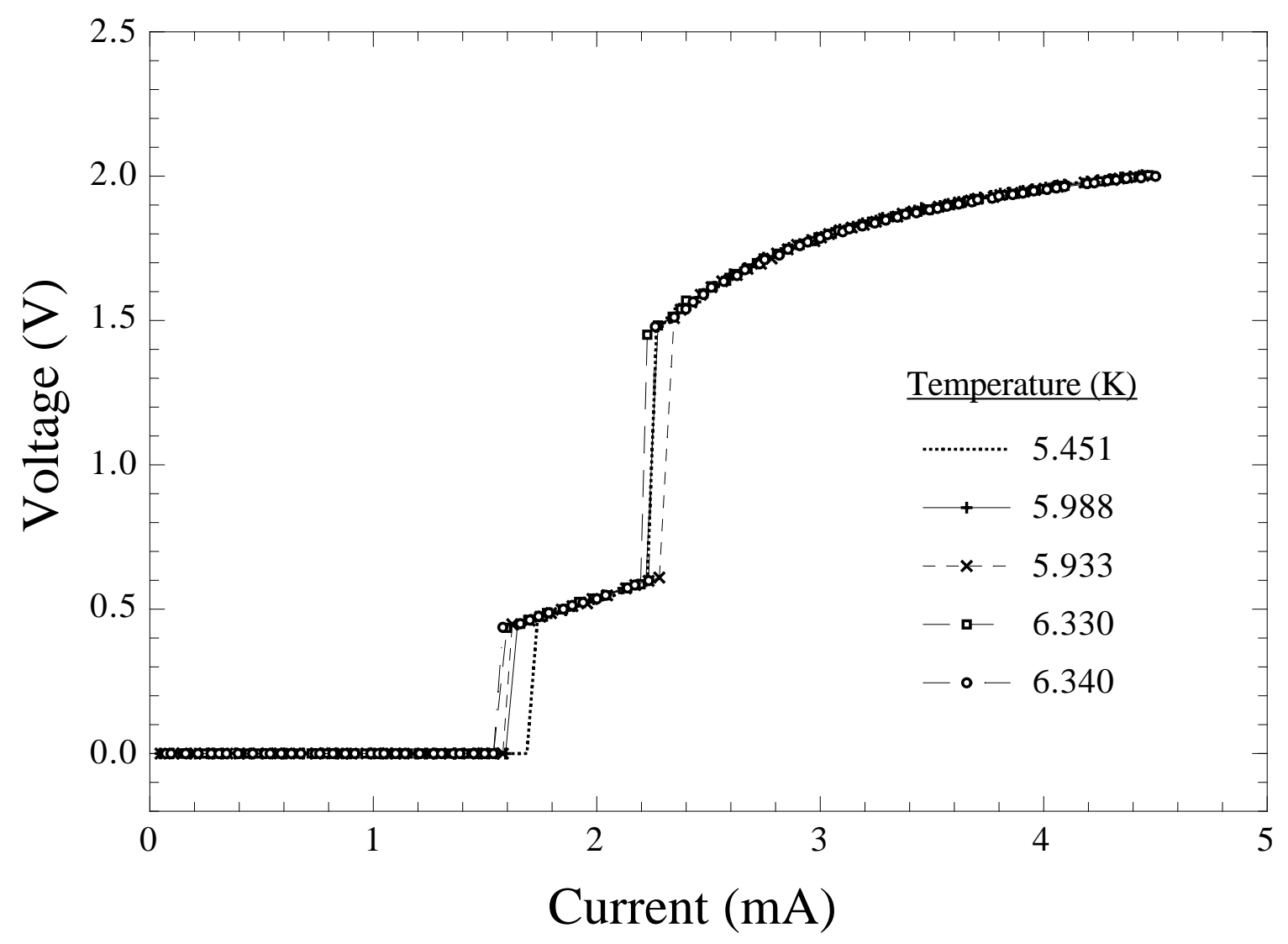

Figure 7 


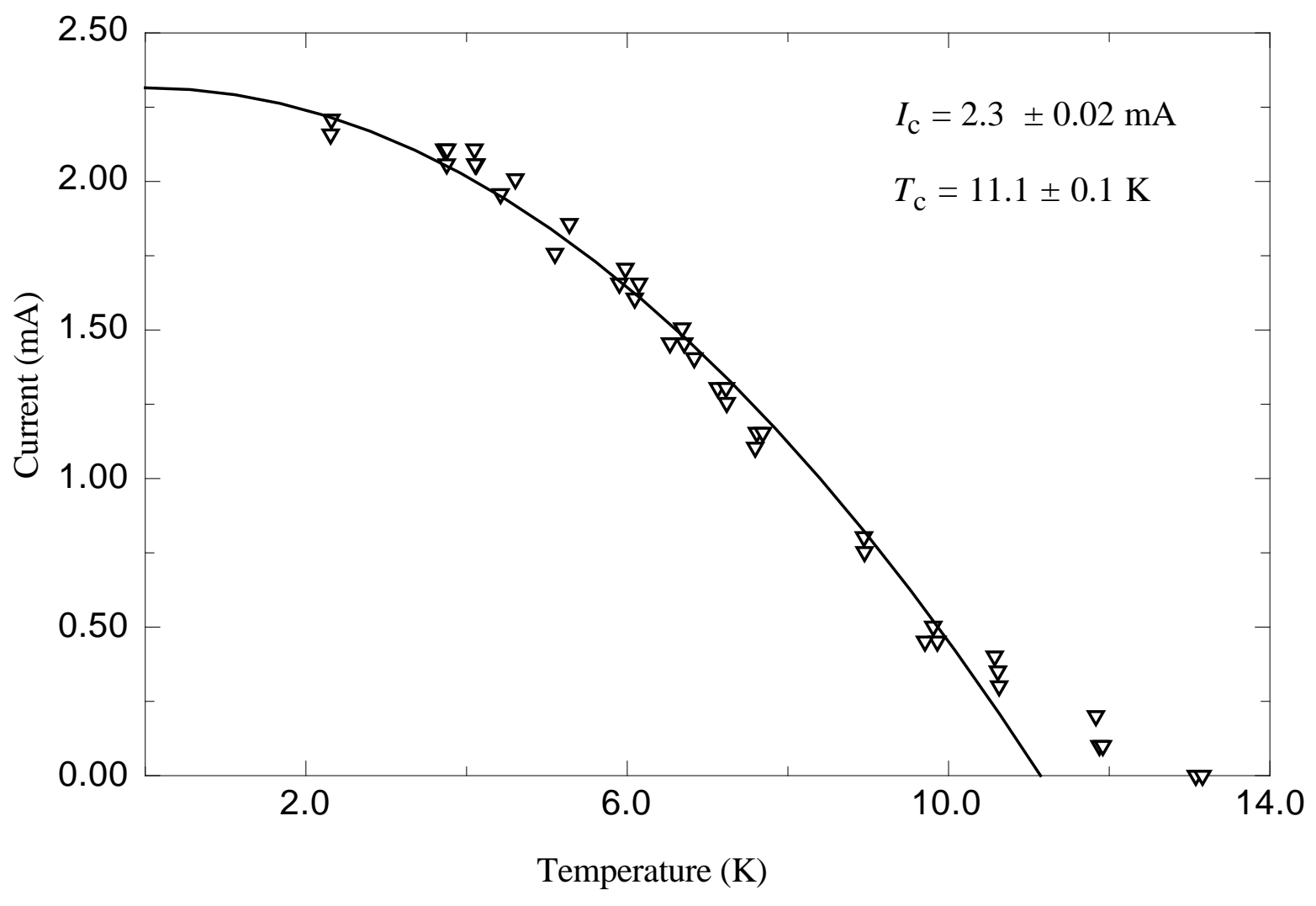

Figure 8 

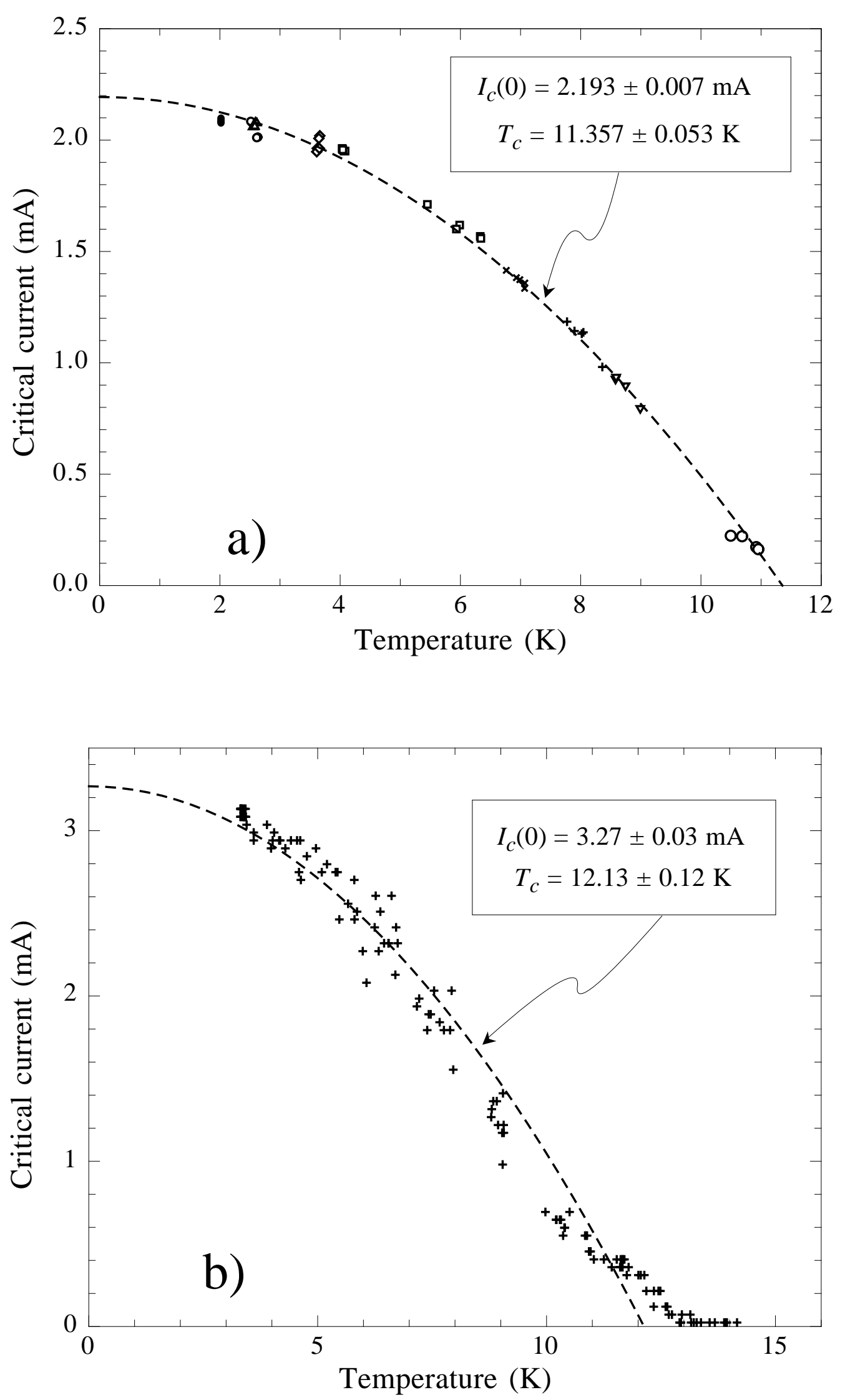

Figure 9 


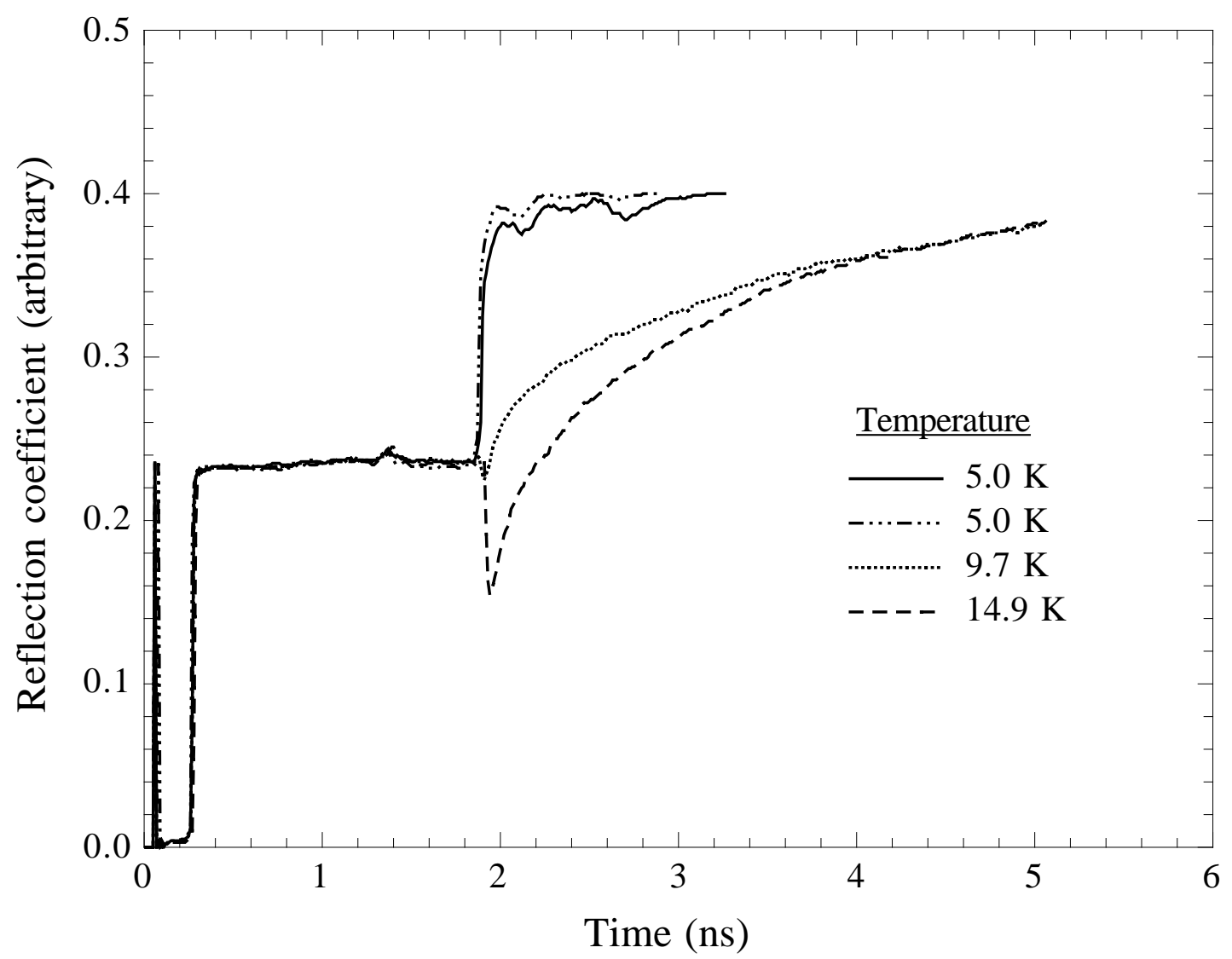

Figure 10

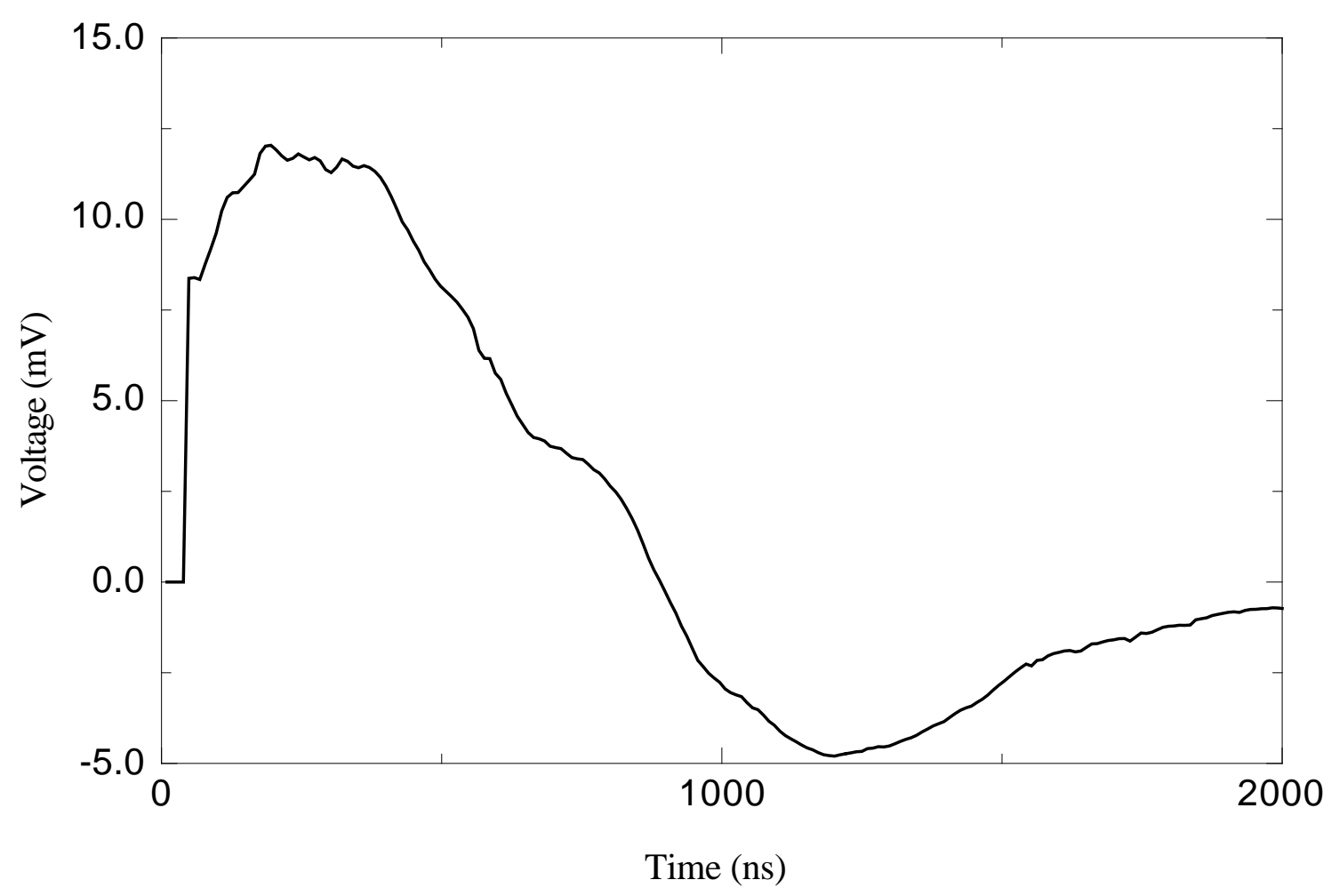

Figure 11 


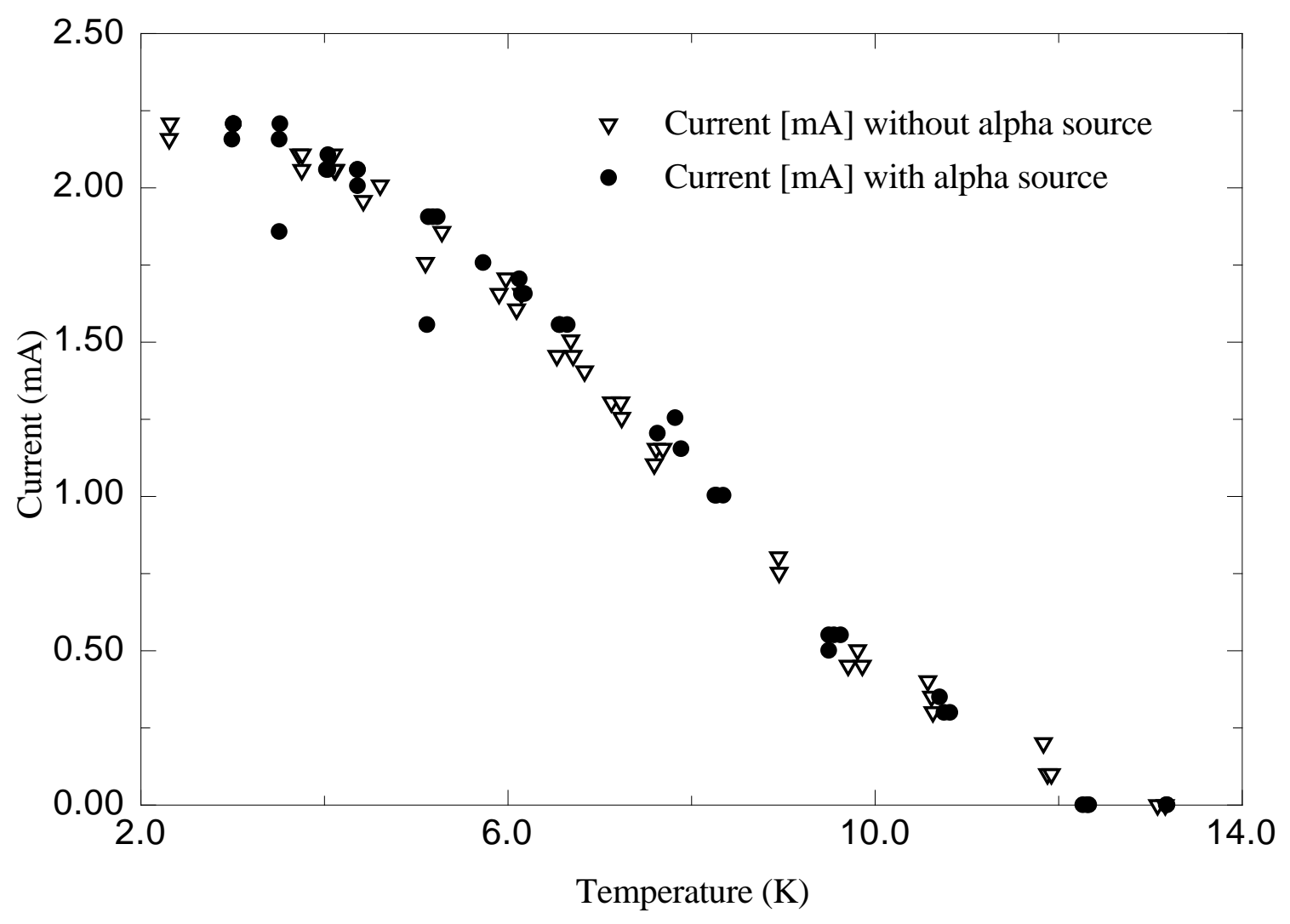

Figure 12 